\title{
Drazin-Star and Star-Drazin Inverses of Bounded Finite Potent Operators on Hilbert Spaces
}

\author{
Fernando Pablos Romo®
}

\begin{abstract}
The aim of this work is to extend to bounded finite potent endomorphisms on arbitrary Hilbert spaces the notions of the Drazin-Star and the Star-Drazin of matrices that have been recently introduced by D. Mosić. The existence, structure and main properties of these operators are given. In particular, we obtain new properties of the Drazin-Star and the Star-Drazin of a finite complex matrix. Moreover, the explicit solutions of some infinite linear systems on Hilbert spaces from the Drazin-Star inverse of a bounded finite potent endomorphism are studied.
\end{abstract}

Mathematics Subject Classification. 46C05, 47L30, 15A09.

Keywords. Bounded operator, Hilbert space, Star-Drazin inverse, DrazinStar inverse.

\section{Introduction}

For an arbitrary $(n \times n)$-matrix $A$ with entries in the complex numbers, the index of $A, i(A) \geq 0$, is the smallest integer such that $\operatorname{rk}\left(A^{i(A)}\right)=\operatorname{rk}\left(A^{i(A)+1}\right)$.

In 1958, Drazin in [8] showed the existence of a unique $n \times n$ complex matrix $A^{D}$, called the Drazin inverse, satisfying the equations:

- $A^{r+1} A^{D}=A^{r}$ for $r=i(A)$;

- $A^{D} A A^{D}=A^{D}$;

- $A^{D} A=A A^{D}$.

When $i(A) \leq 1$, it is known that the Drazin inverse $A^{D}$ coincides with the group inverse $A^{\#}$. 
Moreover, given again $A \in \operatorname{Mat}_{n \times n}(\mathbb{C})$, D. Mosić has introduced in [10] the notions of Drazin-Star and Star-Drazin matrices.

The Drazin-Star of a finite square complex matrix $A$ is $A^{D, *}=A^{D} A A^{*}$ and is defined as the unique solution of the system of equations:

$$
\begin{aligned}
X\left(A^{\dagger}\right)^{*} X & =X \\
A^{r} X & =A^{r} A^{*} \\
X\left(A^{\dagger}\right)^{*} & =A^{D} A,
\end{aligned}
$$

where $i(A)=r, A^{\dagger}$ is the Moore-Penrose inverse of $A$ and $A^{*}$ is the conjugate transpose of $A$.

Analogously, the Star-Drazin of $A$ is the unique solution of

$$
\begin{aligned}
X\left(A^{\dagger}\right)^{*} X & =X \\
X A^{r} & =A^{*} A^{r} \\
\left(A^{\dagger}\right)^{*} X & =A^{D} A
\end{aligned}
$$

and its explicit expression is $A^{*, D}=A^{*} A A^{D}$.

In particular, when $i(A) \leq 1$, the Group-Star of $A$ is the unique matrix $A^{\#, *}$ satisfying that

- $A^{\#, *}\left(A^{\dagger}\right)^{*} A^{\#, *}=A^{\#, *}$;

- $A A^{\#, *}=A A^{*}$;

- $A^{\#, *}\left(A^{\dagger}\right)^{*}=A^{\#} A$; and the Star-Group $A^{*, \#}$ is uniquely determined from the conditions:

- $A^{*, \#}\left(A^{\dagger}\right)^{*} A^{*, \#}=A^{*, \#}$;

- $A^{*, \#} A=A^{*} A$;

- $\left(A^{\dagger}\right)^{*} A^{*, \#}=A^{\#} A$.

On the other hand, the notion of finite potent endomorphism on an arbitrary vector space was introduced by Tate in [20] as a basic tool for his elegant definition of Abstract Residues.

During the last decade, the theory of finite potent endomorphisms have been applied to studying different topics related to Algebra, Arithmetic and Algebraic Geometry. Thus, Yekutieli in [21] and Braunling in [2] and [3] have addressed problems of arithmetic symbols by using properties of finite potent endomorphism; Debry in [7] and Taelman in [19] have offered results about Drinfeld modules from these linear operators; and Cabezas Sánchez and Pablos Romo have given explicit solutions of infinite linear systems from reflexive generalized inverses of finite potent endomorphisms in [4]. Moreover, the author of this work has extended to finite potent endomorphisms the notions of Drazin inverse, Group inverse and DMP inverses in [13], [15] and [16] and, recently, has studied the properties of bounded finite potent operators on Hilbert spaces in [14]. As far as we know, this last paper is the first approach for studying finite potent endomorphisms from the point of view of the Functional Analysis that has appeared in the literature. Furthermore, the properties and the 
structure of the Drazin inverse and the DMP inverses of bounded finite potent operators on Hilbert spaces have been provided in [12].

The aim of this work is to extend to bounded finite potent endomorphisms on arbitrary Hilbert spaces the notions of the Drazin-Star and the Star-Drazin of matrices that have been recently introduced by D. Mosić. The existence, structure and main properties of these operators are given. In particular, we obtain new properties of the Drazin-Star and the Star-Drazin of a finite complex matrix. Moreover, the explicit solutions of some infinite linear systems on Hilbert spaces from the Drazin-Star inverse of a bounded finite potent endomorphism are studied.

The paper is organized as follows. In Sect. 2 we recall the definitions and main properties of finite potent endomorphisms, the Drazin inverse, the corenilpotent decomposition of a finite potent endomorphism, the Moore-Penrose inverse, the bounded finite potent operators and the Drazin-Moore-Penrose inverses.

Section 3 contains the main results of this work. Indeed, in this section we prove the existence and uniqueness of the Drazin-Star and the Star-Drazin inverses of a bounded finite potent operators on Hilbert spaces, we determine the structures of these operators and we give their main properties. Moreover, for bounded finite potent endomorphisms with index less or equal to 1, we study the Group-Star and the Star-Group inverses.

Finally, the purpose of Sect. 4 is to apply the properties of the Drazin-Star inverse of a bounded finite potent endomorphism for studying the consistence and the general solutions of linear systems on Hilbert spaces.

\section{Preliminaries}

This section is added for the sake of completeness.

\subsection{Finite Potent Endomorphisms}

Let $k$ be an arbitrary field, and let $V$ be a $k$-vector space.

Let us now consider an endomorphism $\varphi$ of $V$. We say that $\varphi$ is "finite potent" if $\varphi^{n} V$ is finite dimensional for some $n$. This definition was introduced by J. Tate in [20] as a basic tool for his elegant definition of Abstract Residues.

In 2007, Argerami et al. showed in [1] that an endomorphism $\varphi$ is finite potent if and only if $V$ admits a $\varphi$-invariant decomposition $V=U_{\varphi} \oplus W_{\varphi}$ such that $\varphi_{\left.\right|_{U_{\varphi}}}$ is nilpotent, $W_{\varphi}$ is finite dimensional, and $\varphi_{\left.\right|_{W_{\varphi}}}: W_{\varphi} \stackrel{\sim}{\longrightarrow} W_{\varphi}$ is an isomorphism.

Indeed, if $k[x]$ is the algebra of polynomials in the variable $x$ with coefficients in $k$, we may view $V$ as an $k[x]$-module via $\varphi$, and the explicit definition of the above $\varphi$-invariant subspaces of $V$ is:

$$
\text { - } U_{\varphi}=\left\{v \in V \text { such that } \varphi^{m}(v)=0 \text { for some } m\right\} \text {. }
$$


- $W_{\varphi}=\left\{\begin{array}{l}v \in V \text { such that } p(\varphi)(v)=0 \text { for some } p(x) \in k[x] \\ \text { relatively prime to } x\end{array}\right\}$.

Note that if the annihilator polynomial of $\varphi$ is $x^{m} \cdot p(x)$ with $(x, p(x))=1$, then $U_{\varphi}=\operatorname{Ker} \varphi^{m}$ and $W_{\varphi}=\operatorname{Ker} p(\varphi)$.

Hence, this decomposition is unique. We shall call this decomposition the $\varphi$-invariant AST-decomposition of $V$.

Basic examples of finite potent endomorphisms are all endomorphisms of a finite-dimensional vector space and finite rank or nilpotent endomorphisms of infinite-dimensional vector spaces.

For a finite potent endomorphism $\varphi$, a trace $\operatorname{Tr}_{V}(\varphi) \in k$ may be defined as $\operatorname{Tr}_{V}(\varphi)=\operatorname{Tr}_{W_{\varphi}}\left(\varphi_{\left.\right|_{W_{\varphi}}}\right)$.

This trace has the following properties:

(1) if $V$ is finite dimensional, then $\operatorname{Tr}_{V}(\varphi)$ is the ordinary trace;

(2) if $W$ is a subspace of $V$ such that $\varphi W \subset W$, then

$$
\operatorname{Tr}_{V}(\varphi)=\operatorname{Tr}_{W}(\varphi)+\operatorname{Tr}_{V / W}(\varphi)
$$

(3) if $\varphi$ is nilpotent, then $\operatorname{Tr}_{V}(\varphi)=0$.

Usually, $\operatorname{Tr}_{V}$ is named "Tate's trace".

Moreover, Hernández Serrano and the author of this paper have offered in [9] a definition of a determinant for finite potent endomorphisms satisfying the following properties:

- if $V$ is finite dimensional, then $\operatorname{det}_{V}^{k}(1+\varphi)$ is the ordinary determinant;

- if $W$ is a subspace of $V$ such that $\varphi W \subset W$, then

$$
\operatorname{det}_{V}^{k}(1+\varphi)=\operatorname{det}_{W}^{k}(1+\varphi) \cdot \operatorname{det}_{V / W}^{k}(1+\varphi)
$$

- if $\varphi$ is nilpotent, then $\operatorname{det}_{V}^{k}(1+\varphi)=1$.

For details readers are referred to [9], [17], [18] and [20].

\subsection{Drazin Inverse of Finite Potent Endomorphisms}

2.2.1. Drazin Inverse of $(\boldsymbol{n} \times \boldsymbol{n})$-Matrices. Let $A \in \operatorname{Mat}_{n \times n}(\mathbb{C})$.

Definition 2.1. The "index of $A$ ", $i(A) \geq 0$, is the smallest integer such that $\operatorname{rk}\left(A^{i(A)}\right)=\operatorname{rk}\left(A^{i(A)+1}\right)$.

In 1958, given a matrix $A \in \operatorname{Mat}_{n \times n}(\mathbb{C})$ with $i(A)=k$, Drazin [8] showed the existence of a unique $(n \times n)$-matrix $A^{D}$ satisfying the equations:

- $A^{k+1} A^{D}=A^{k}$ for $k=i(A)$;

- $A^{D} A A^{D}=A^{D}$;

- $A^{D} A=A A^{D}$.

The Drazin inverse $A^{D}$ also verifies that

- $\left(A^{D}\right)^{D}=A$ if and only if $i(A) \leq 1$;

- if $A^{2}=A$, then $A^{D}=A$. 
2.2.2. Drazin Inverse of Finite Potent Endomorphisms. Let $V$ be an arbitrary $k$-vector space and let $\varphi \in \operatorname{End}_{k}(V)$ be a finite potent endomorphism of $V$. Let us consider the AST-decomposition $V=U_{\varphi} \oplus W_{\varphi}$ induced by $\varphi$.

We shall call "index of $\varphi$ ", $i(\varphi)$, to the nilpotent order of $\varphi_{\left.\right|_{U}}$, which coincides with the smaller $n \in \mathbb{N}$ such that $\operatorname{Im} \varphi^{n}=W_{\varphi}$. One has that $i(\varphi)=0$ if and only if $V$ is a finite-dimensional vector space and $\varphi$ is an automorphism. In [16, Lemma 3.2] is proved that for finite-dimensional vector spaces, this definition of index coincides with Definition 2.1 for matrices associated with endomorphisms of finite-dimensional vector spaces.

For each finite potent endomorphism $\varphi$ there exists a unique finite potent endomorphism $\varphi^{D}$ that satisfies that:

(1) $\varphi^{r+1} \circ \varphi^{D}=\varphi^{r}$;

(2) $\varphi^{D} \circ \varphi \circ \varphi^{D}=\varphi^{D}$;

(3) $\varphi^{D} \circ \varphi=\varphi \circ \varphi^{D}$,

where $r$ is the index of $\varphi$.

The map $\varphi^{D}$ is the Drazin inverse of $\varphi$ and is the unique linear map such that

$$
\varphi^{D}(v)= \begin{cases}\left(\varphi_{\left.\right|_{W_{\varphi}}}\right)^{-1} & \text { if } v \in W_{\varphi} \\ 0 & \text { if } v \in U_{\varphi}\end{cases}
$$

Moreover, $\varphi^{D}$ satisfies the following properties:

- $\left(\varphi^{D}\right)^{D}=\varphi$ if and only if $i(\varphi) \leq 1$;

- $\varphi=\varphi^{D}$ if and only if $\varphi_{\left.\right|_{\varphi}}=0$ and $\left(\varphi_{\left.\right|_{W_{\varphi}}}\right)^{2}=\operatorname{Id}_{\left.\right|_{W_{\varphi}}}$;

- if $\psi$ is a projection finite potent endomorphism, then $\psi^{D}=\psi$.

\subsection{CN Decomposition of a Finite Potent Endomorphism}

Let $V$ be again an arbitrary $k$-vector space. Given a finite potent endomorphism $\varphi \in \operatorname{End}_{k}(V)$, there exists a unique decomposition $\varphi=\varphi_{1}+\varphi_{2}$, where $\varphi_{1}, \varphi_{2} \in \operatorname{End}_{k}(V)$ are finite potent endomorphisms satisfying that

- $i\left(\varphi_{1}\right) \leq 1$

- $\varphi_{2}$ is nilpotent;

- $\varphi_{1} \circ \varphi_{2}=\varphi_{2} \circ \varphi_{1}=0$.

According to [11, Theorem 3.2], if $\varphi^{D}$ is the Drazin inverse of $\varphi$, one has that $\varphi_{1}=\varphi \circ \varphi^{D} \circ \varphi$ is the core part of $\varphi$. Also, $\varphi_{2}$ is named the nilpotent part of $\varphi$ and one has that

$$
\varphi=\varphi_{1} \Longleftrightarrow U_{\varphi}=\operatorname{Ker} \varphi \Longleftrightarrow W_{\varphi}=\operatorname{Im} \varphi \Longleftrightarrow\left(\varphi^{D}\right)^{D}=\varphi \Longleftrightarrow i(\varphi) \leq 1 \text {. }
$$

Moreover, if $V=W_{\varphi} \oplus U_{\varphi}$ is the AST-decomposition of $V$ induced by $\varphi$, then $\varphi_{1}$ and $\varphi_{2}$ are the unique linear maps such that

$$
\varphi_{1}(v)=\left\{\begin{array}{ll}
\varphi(v) & \text { if } v \in W_{\varphi} \\
0 & \text { if } v \in U_{\varphi}
\end{array} \quad \text { and } \quad \varphi_{2}(v)= \begin{cases}0 & \text { if } v \in W_{\varphi} \\
\varphi(v) & \text { if } v \in U_{\varphi}\end{cases}\right.
$$


By definition of Tate's trace, for every finite potent endomorphism $\varphi \in$ $\operatorname{End}_{k}(V)$, one has that

$$
\operatorname{Tr}_{V}(\varphi)=\operatorname{Tr}_{V}\left(\varphi_{1}\right)
$$

\subsection{Bounded Finite Potent Endomorphisms on Hilbert Spaces}

Let $k$ be the field of the real numbers or the field of the complex numbers, and let $V$ be a $k$-vector space.

An inner product on $V$ is a map $g: V \times V \rightarrow k$ satisfying:

- $g$ is linear in its first argument:

$$
g\left(\lambda v_{1}+\mu v_{2}, v^{\prime}\right)=\lambda g\left(v_{1}, v^{\prime}\right)+\mu g\left(v_{2}, v^{\prime}\right) \text { for every } v_{1}, v_{2}, v^{\prime} \in V
$$

- $g\left(v^{\prime}, v\right)=\overline{g\left(v, v^{\prime}\right)}$ for all $v, v^{\prime} \in V$, where $\overline{g\left(v, v^{\prime}\right)}$ is the complex conjugate of $g\left(v, v^{\prime}\right)$;

- $g$ is positive definite:

$$
g(v, v) \geq 0 \text { and } g(v, v)=0 \Longleftrightarrow v=0 .
$$

Note that $g(v, v) \in \mathbb{R}$ for each $v \in V$, because $g(v, v)=\overline{g(v, v)}$.

An inner product space is a pair $(V, g)$.

If $(V, g)$ is an inner product vector space, we say that two vectors $v, v^{\prime} \in V$ are orthogonal when $g\left(v, v^{\prime}\right)=0=g\left(v^{\prime}, v\right)$. Also, given a subspace $L$ of an inner vector space $(V, g)$, we shall call "orthogonal of $L^{\prime}, L^{\perp}$, to the subset of $V$ consists of the vectors that are orthogonal to every $h \in L$, that is

$$
L^{\perp}=\{v \in V \text { such that } g(v, h)=0 \text { for every } h \in L\} .
$$

The norm on an inner product space $(V, g)$ is the real-valued function

$$
\begin{aligned}
\|\cdot\|_{g}: V & \longrightarrow \mathbb{R} \\
v & \longmapsto+\sqrt{g(v, v),}
\end{aligned}
$$

and the distance is the map

$$
\begin{aligned}
d_{g}: V \times V & \longrightarrow \mathbb{R} \\
\left(v, v^{\prime}\right) & \longmapsto\left\|v^{\prime}-v\right\|_{g} .
\end{aligned}
$$

Every inner product vector space $(V, g)$ has a natural structure of metric topological space determined by the distance $d_{g}$. Complete inner product $\mathbb{C}$ vector spaces are known as "Hilbert spaces". Usually, the inner product of a Hilbert space $\mathcal{H}$ is denoted by $\langle\cdot, \cdot\rangle_{\mathcal{H}}$. Henceforth, we shall write $\mathcal{H}$ to refer to a Hilbert space and keep the inner product $<\cdot, \cdot\rangle_{\mathcal{H}}$ implicit.

If $L \subseteq \mathcal{H}$ is a subspace of an arbitrary Hilbert space, it is known that $\left(L^{\perp}\right)^{\perp}=\bar{L}$ where $\bar{L}$ denotes the closure of $L$. Accordingly, if $L \subseteq \mathcal{H}$ is closed, then $\left(L^{\perp}\right)^{\perp}=L$ and $\mathcal{H}=L \oplus L^{\perp}$.

We shall now recall the main properties of bounded operators of Hilbert spaces. 
Definition 2.2. If $\mathcal{H}_{1}$ and $\mathcal{H}_{2}$ are two Hilbert spaces, a linear map $f: \mathcal{H}_{1} \rightarrow \mathcal{H}_{2}$ is said "bounded" when there exists $C \in \mathbb{R}^{+}$such that

$$
\|f(v)\|_{g_{2}} \leq C \cdot\|v\|_{g_{1}}
$$

for every $v \in \mathcal{H}_{1}$.

We shall denote by $B\left(\mathcal{H}_{1}, \mathcal{H}_{2}\right)$ the set of bounded linear maps $f: \mathcal{H}_{1} \rightarrow \mathcal{H}_{2}$ and by $B(\mathcal{H})$ the set of bounded endomorphisms of a Hilbert space $\mathcal{H}$. Given a linear map $f \in B\left(\mathcal{H}_{1}, \mathcal{H}_{2}\right)$, it is known that $f$ is continuous if and only if $f$ is bounded.

The spectrum of a bounded operator $f \in B(\mathcal{H})$ consists of complex numbers $\lambda$ such that $f-\lambda$ Id is not invertible. We shall denote the spectrum of $f$ by $\sigma(f)$ and it is clear that every eigenvalue of $f$ is an element of $\sigma(f)$. It is known that it is possible that an element of $\sigma(f)$ is not an eigenvalue.

Recently, the author of this work has studied in [14] the set of bounded finite potent endomorphisms on an arbitrary Hilbert space, which will be denoted by $B_{f p}(\mathcal{H})$.

If $\varphi \in B_{f p}(\mathcal{H}), \mathcal{H}=W_{\varphi} \oplus U_{\varphi}$ is the AST-decomposition induced by $\varphi$ and $\varphi=\varphi_{1}+\varphi_{2}$ is the $\mathrm{CN}$-decomposition, then the following properties hold:

(1) $\varphi$ is quasi-compact;

(2) $\varphi_{1}, \varphi_{2} \in B_{f p}(\mathcal{H})$ and $\varphi_{1}$ is of trace class;

(3) $\varphi$ is compact if and only if $\varphi_{2}$ is compact;

(4) if $\operatorname{Tr}\left(\varphi_{1}\right)$ is the trace of $\varphi_{1}$ as a trace class operator, then $\operatorname{Tr}\left(\varphi_{1}\right)=$ $\operatorname{Tr}_{\mathcal{H}}(\varphi)$

(5) given a non-zero $\lambda \in \mathbb{C}$, one has that $\lambda$ is an eigenvalue of $\varphi$ if and only if $\lambda$ is an eigenvalue of $\varphi_{\left.\right|_{W_{\varphi}}}$;

(6) if $i(\varphi) \geq 1$, then $\sigma(\varphi)=\left\{0, \lambda_{1}, \ldots, \lambda_{n}\right\}$ where $\left\{\lambda_{1}, \ldots, \lambda_{n}\right\}$ are the eigenvalues of $\varphi_{\left.\right|_{W_{\varphi}}}$;

(7) $\operatorname{Tr}_{\mathcal{H}}(\varphi)=\operatorname{Tr}_{W_{\varphi}}\left(\varphi_{\left.\right|_{W_{\varphi}}}\right)=\operatorname{Tr}\left(\varphi_{1}\right)=\operatorname{Tr}_{\mathcal{H}}^{L}(\varphi)=\operatorname{Tr}_{\mathcal{H}}^{R}(\varphi)$,

where $\operatorname{Tr}_{\mathcal{H}}(\varphi)$ is the Tate's trace of $\varphi$ as a finite potent endomorphism; $\operatorname{Tr}_{W_{\varphi}}\left(\varphi_{\left.\right|_{W_{\varphi}}}\right)$ is the trace of the endomorphism $\varphi_{\left.\right|_{W_{\varphi}}}$ on the finite-dimensional $\mathbb{C}$-vector space $W_{\varphi} ; \operatorname{Tr}\left(\varphi_{1}\right)$ is the trace of $\varphi_{1}$ of a trace class operator; $\operatorname{Tr}_{\mathcal{H}}^{L}(\varphi)$ is the Leray trace and $\operatorname{Tr}_{\mathcal{H}}^{R}(\varphi)$ is the trace of $\varphi$ as a Riesz trace class operator.

Moreover, the adjoint operator $\varphi^{*}$ satisfies that:

(1) $\varphi^{*} \in B_{f p}(\mathcal{H})$;

(2) $i\left(\varphi^{*}\right)=i(\varphi)$

(3) $\varphi^{*}=\left(\varphi_{1}\right)^{*}+\left(\varphi_{2}\right)^{*}$ is the $\mathrm{CN}$-decomposition of $\varphi^{*}$;

(4) if $\mathcal{H}=W_{\varphi^{*}} \oplus U_{\varphi^{*}}$ is the AST-decomposition induced by $\varphi^{*}$, then one has that $W_{\varphi^{*}}=\left[U_{\varphi}\right]^{\perp}$ and $U_{\varphi^{*}}=\left[W_{\varphi}\right]^{\perp}$;

(5) $\sigma\left(\varphi^{*}\right)=\overline{\sigma(\varphi)}$.

Now we recall a statement of [14] that shall be useful for the present work. 
Thus, it follows from [14, Proposition 4.1] that

Proposition 2.3. If $\mathcal{H}$ is a Hilbert space and we consider $\varphi \in B_{f p}(\mathcal{H})$, then the adjoint $\varphi^{*}$ is also a bounded finite potent endomorphism.

2.4.1. Drazin Inverse of a Bounded Finite Potent Operator. Our task is now to recall different properties of the Drazin inverse of a bounded finite potent endomorphism on a Hilbert space offered in [12]. Thus, if $\mathcal{H}$ is a Hilbert space and $\varphi \in B_{f p}(\mathcal{H})$, then the Drazin inverse $\varphi^{D}$ is also a bounded finite potent endomorphism satisfying that

(1) $\left(\varphi^{D}\right)^{*}=\left(\varphi^{*}\right)^{D}$;

(2) $\left(\left[\varphi^{*}\right]^{D}\right)^{D}=\varphi^{*}$ if and only if $i(\varphi) \leq 1$;

(3) $\varphi^{*}=\left(\varphi^{*}\right)^{D}$ if and only if $\varphi_{\left.\right|_{U_{\varphi}}}=0$ and $\left(\varphi_{\left.\right|_{W_{\varphi}}}\right)^{2}=\operatorname{Id}_{\left.\right|_{W_{\varphi}}}$;

(4) if $\psi$ is a projection finite potent endomorphism, then $\left(\psi^{*}\right)^{D}=\psi^{*}$.

\subsection{Moore-Penrose Inverse}

2.5.1. Moore-Penrose Inverse of an $(n \times m)$-Matrix. Let $\mathbb{C}$ be the field of complex number. Given a matrix $A \in \operatorname{Mat}_{n \times m}(\mathbb{C})$, the Moore-Penrose inverse of $A$ is a matrix $A^{\dagger} \in \operatorname{Mat}_{m \times n}(\mathbb{C})$ such that

- $A A^{\dagger} A=A$;

- $A^{\dagger} A A^{\dagger}=A^{\dagger}$

- $\left(A A^{\dagger}\right)^{*}=A A^{\dagger}$;

- $\left(A^{\dagger} A\right)^{*}=A^{\dagger} A$;

$B^{*}$ being the conjugate transpose of the matrix $B$.

The Moore-Penrose inverse of $A$ always exists, it is unique, $\left[A^{\dagger}\right]^{\dagger}=A$, and, if $A \in \mathbb{C}^{n \times n}$ is non-singular, then the Moore-Penrose inverse of $A$ coincides with the inverse matrix $A^{-1}$.

For details, readers are referred to [6].

2.5.2. Moore-Penrose Inverse of a Linear Map Over Arbitrary Inner Product Spaces. Let $(V, g)$ and $(W, \bar{g})$ be inner product vector spaces over $k$, with $k=\mathbb{C}$ or $k=\mathbb{R}$.

Given a linear map $f: V \rightarrow W$, a linear map $f^{+}: W \rightarrow V$ is a reflexive generalized inverse of $f$ when

- $f \circ f^{+} \circ f=f$

- $f^{+} \circ f \circ f^{+}=f^{+}$.

Definition 2.4. Given a linear map $f: V \rightarrow W$, we say that $f$ is admissible for the Moore-Penrose inverse when $V=\operatorname{Ker} f \oplus[\operatorname{Ker} f]^{\perp}$ and $W=\operatorname{Im} f \oplus$ $[\operatorname{Im} f]^{\perp}$.

According to [5, Theorem 3.12], if $(V, g)$ and $(W, \bar{g})$ are inner product spaces over $k$, then $f: V \rightarrow W$ is a linear map admissible for the MoorePenrose inverse if and only if there exists a unique linear map $f^{\dagger}: W \rightarrow V$ such that 
(1) $f^{\dagger}$ is a reflexive generalized inverse of $f$;

(2) $f^{\dagger} \circ f$ and $f \circ f^{\dagger}$ are self-adjoint, that is

- $g\left(\left[f^{\dagger} \circ f\right](v), v^{\prime}\right)=g\left(v,\left[f^{\dagger} \circ f\right]\left(v^{\prime}\right)\right.$;

- $\bar{g}\left(\left[f \circ f^{\dagger}\right](w), w^{\prime}\right)=\bar{g}\left(w,\left[f \circ f^{\dagger}\right]\left(w^{\prime}\right)\right.$;

for all $v, v^{\prime} \in V$ and $w, w^{\prime} \in W$. The operator $f^{\dagger}$ is named the MoorePenrose inverse of $f$ and it is the unique linear map satisfying that

$$
f^{\dagger}(w)= \begin{cases}\left(f_{\left.\right|_{[\operatorname{Ker} f]^{\perp}}}\right)^{-1}(w) & \text { if } w \in \operatorname{Im} f \\ 0 & \text { if } w \in[\operatorname{Im} f]^{\perp} .\end{cases}
$$

The Moore-Penrose inverse $f^{\dagger}: W \rightarrow V$ also satisfies the following properties:

- $f^{\dagger}$ is admissible for the Moore-Penrose inverse and $\left(f^{\dagger}\right)^{\dagger}=f$;

- If $f \in \operatorname{End}_{k}(V)$ and $f$ is an isomorphism, then $f^{\dagger}=f^{-1}$;

- $f^{\dagger} \circ f=P_{[\operatorname{Ker} f]^{\perp}}$;

- $f \circ f^{\dagger}=P_{\operatorname{Im} f}$,

where $P_{[\operatorname{Ker} f]^{\perp}}$ and $P_{\operatorname{Im} f}$ are the projections induced by the decompositions $V=\operatorname{Ker} f \oplus[\operatorname{Ker} f]^{\perp}$ and $W=\operatorname{Im} f \oplus[\operatorname{Im} f]^{\perp}$ respectively.

Lemma 2.5. If $V$ is a k-vector space, $f \in \operatorname{End}_{k}(V)$ is an endomorphism admissible for the Moore-Penrose inverse and $g \in \operatorname{End}_{k}(V)$ is such that $\operatorname{Im} g \subseteq \operatorname{Im} f$, then

$$
f \circ f^{\dagger} \circ g=g
$$

Proof. The statement is immediately deduced from the equality $f \circ f^{\dagger}=P_{\operatorname{Im} f}$.

Analogously, we can easily check that

Lemma 2.6. If $V$ is a k-vector space, $f \in \operatorname{End}_{k}(V)$ is an endomorphism admissible for the Moore-Penrose inverse and $\tilde{g} \in \operatorname{End}_{k}(V)$ is such that Im $\tilde{g} \subseteq[\operatorname{Ker} f]^{\perp}$, then

$$
f^{\dagger} \circ f \circ \tilde{g}=\tilde{g}
$$

Readers are referred to [5] for more details on the Moore-Penrose inverse over arbitrary inner product spaces.

2.5.3. Moore-Penrose Inverse of a Bounded Linear Map. We shall now recall different properties of the Moore-Penrose inverse of a bounded linear map between two Hilbert spaces.

Let $\mathcal{H}_{1}$ and $\mathcal{H}_{2}$ be two Hilbert spaces. Given a linear map $f: \mathcal{H}_{1} \rightarrow \mathcal{H}_{2}$, recall from Definition 2.4 that $f$ is admissible for the Moore-Penrose inverse when $\mathcal{H}_{1}=\operatorname{Ker} f \oplus[\operatorname{Ker} f]^{\perp}$ and $\mathcal{H}_{2}=\operatorname{Im} f \oplus[\operatorname{Im} f]^{\perp}$.

If $f \in B\left(\mathcal{H}_{1}, \mathcal{H}_{2}\right)$, it is known that $f$ is admissible for the Moore-Penrose inverse if and only if $\operatorname{Im} f$ is a closed subspace of $\mathcal{H}_{1}$. Also, if $f \in B\left(\mathcal{H}_{1}, \mathcal{H}_{2}\right)$ is admissible for the Moore-Penrose inverse, then $f^{\dagger} \in B\left(\mathcal{H}_{2}, \mathcal{H}_{1}\right)$.

Moreover, [12, Proposition 3.3] shows that 
Proposition 2.7. If $f \in B\left(\mathcal{H}_{1}, \mathcal{H}_{2}\right)$ is admissible for the Moore-Penrose inverse, then $f^{*}$ is also admissible for the Moore-Penrose inverse and $\left(f^{*}\right)^{\dagger}=$ $\left(f^{\dagger}\right)^{*}$.

Furthermore, from the properties of the Moore-Penrose inverse of a linear map and different statements proved in [12], one has that

Lemma 2.8. If $f \in B\left(\mathcal{H}_{1}, \mathcal{H}_{2}\right)$ such that Im $f$ is a closed subspace of $\mathcal{H}_{2}$, then the following assertions hold:

- Im $f^{*}$ is also a closed subspace of $\mathcal{H}_{1}$;

- $f^{*} \circ\left(f^{*}\right)^{\dagger}=P_{[\operatorname{Ker} f]^{\perp}}$;

- $\left(f^{*}\right)^{\dagger} \circ f^{*}=P_{\operatorname{Im} f}$;

- $f^{*} \circ f \circ f^{\dagger}=f^{*}$;

- $f^{\dagger} \circ f \circ f^{*}=f^{*}$;

- $\left(f^{*}\right)^{\dagger} \circ f^{*} \circ f=f$;

- $f \circ f^{*} \circ\left(f^{*}\right)^{\dagger}=f$,

where $P_{[\operatorname{Ker} f]^{\perp}}$ and $P_{\operatorname{Im} f}$ are the projections induced by the decompositions $\mathcal{H}_{1}=\operatorname{Ker} f \oplus[\operatorname{Ker} f]^{\perp}$ and $\mathcal{H}_{2}=\operatorname{Im} f \oplus[\operatorname{Im} f]^{\perp}$ respectively.

\subsubsection{Drazin-Moore-Penrose Inverses of a Bounded Finite Potent Operator.}

To finish this section we provide the main properties of the Drazin-MoorePenrose inverse of a bounded finite potent operator on a Hilbert space.

Given an arbitrary $k$-vector space $V$ and a finite potent endomorphism $\varphi \in \operatorname{End}_{k}(V)$ admissible for the Moore-Penrose inverse, according to [15, Theorem 3.2], there exists a unique finite potent endomorphism $\varphi^{d, \dagger} \in \operatorname{End}_{k}(V)$ verifying that

(1) $\varphi^{d, \dagger} \circ \varphi \circ \varphi^{d, \dagger}=\varphi^{d, \dagger}$;

(2) $\varphi^{r} \circ \varphi^{d, \dagger}=\varphi^{r} \circ \varphi^{\dagger}$ with $r=i(\varphi)$;

(3) $\varphi^{d, \dagger} \circ \varphi=\varphi^{D} \circ \varphi$,

where $\varphi^{D}$ is the Drazin inverse and $\varphi^{\dagger}$ is the Moore-Penrose inverse of $\varphi$.

The map $\varphi^{d, \dagger}$ is called the left-Drazin Moore-Penrose (IDMP) inverse of $\varphi$.

Moreover, from [15, Theorem 3.17] one has the existence and uniqueness of a finite potent endomorphism $\varphi^{\dagger, d} \in \operatorname{End}_{k}(V)$ satisfying that

(1) $\varphi^{\dagger, d} \circ \varphi \circ \varphi^{\dagger, d}=\varphi^{\dagger, d}$;

(2) $\varphi^{\dagger, d} \circ \varphi^{r}=\varphi^{\dagger} \circ \varphi^{r}$ with $r=i(\varphi)$;

(3) $\varphi \circ \varphi^{\dagger, d}=\varphi \circ \varphi^{D}$.

The map $\varphi^{\dagger, d}$ is the right-Drazin Moore-Penrose (rDMP) inverse of $\varphi$.

Let us again consider a Hilbert space $\mathcal{H}$ and an operator $\varphi \in B_{f p}(\mathcal{H})$ with closed $\operatorname{Im} \varphi$. If $\varphi^{*}$ is the adjoint of $\varphi$ and $\varphi=\varphi_{1}+\varphi_{2}$ is again the $\mathrm{CN}$-decomposition of $\varphi$, from the statements of [12] it is known that

- $\varphi^{d, \dagger}, \varphi^{\dagger, d} \in B_{f p}(\mathcal{H})$; 
- $\left(\varphi^{*}\right)^{d, \dagger}$ and $\left(\varphi^{*}\right)^{\dagger, d}$ exist, $\left(\varphi^{*}\right)^{d, \dagger},\left(\varphi^{*}\right)^{\dagger, d} \in B_{f p}(\mathcal{H}),\left(\varphi^{*}\right)^{d, \dagger}=\left(\varphi^{\dagger, d}\right)^{*}$ and $\left(\varphi^{*}\right)^{\dagger, d}=\left(\varphi^{d, \dagger}\right)^{*}$

- $\varphi^{*} \circ\left(\varphi^{*}\right)^{d, \dagger} \circ \varphi^{*}=\varphi^{*} \circ\left(\varphi^{*}\right)^{\dagger, d} \circ \varphi^{*}=\left(\varphi_{1}\right)^{*}$;

- $\left(\varphi^{*}\right)^{\dagger, d}=P_{\operatorname{Im} \varphi^{\circ}}\left(\varphi^{*}\right)^{D}$

- $\left(\varphi^{*}\right)^{d, \dagger}=\left(\varphi^{*}\right)^{D} \circ P_{[\operatorname{Ker} \varphi]^{\perp}}$;

- $\left(\left(\left(\varphi^{*}\right)^{d, \dagger}\right)^{D}\right)^{D}=\left(\varphi^{\dagger, d}\right)^{*}$;

- $\left(\left(\left(\varphi^{*}\right)^{\dagger, d}\right)^{D}\right)^{D}=\left(\varphi^{d, \dagger}\right)^{*}$;

- If $\left(\varphi^{*}\right)^{\dagger, d}=\varphi^{*}$ or $\left(\varphi^{*}\right)^{d, \dagger}=\varphi^{*}$, then $\varphi^{\dagger}=\varphi^{D}$;

- $\left(\varphi^{*}\right)^{\dagger, d}=\varphi^{*}=\left(\varphi^{*}\right)^{d, \dagger}$ if and only if $\varphi$ is EP and tripotent;

- $\left(\varphi^{*}\right)^{\dagger, d}=0=\left(\varphi^{*}\right)^{d, \dagger}$ if and only if $\varphi$ is nilpotent or $\varphi=0$;

- $\left(\varphi^{*}\right)^{\dagger, d}=\left(\varphi^{D}\right)^{*}$ if and only if $\operatorname{Ker} \varphi^{\dagger} \subseteq \operatorname{Ker} \varphi^{D}$;

- $\left(\varphi^{*}\right)^{d, \dagger}=\left(\varphi^{D}\right)^{*}$ if and only if $W_{\varphi} \subseteq[\operatorname{Ker} \varphi]^{\perp}$.

\section{Drazin-Star and Star-Drazin Inverses of Bounded Finite Potent Endomorphisms}

Recently, D. Mosić has introduced in [10] the notions of Drazin-Star and Star-Drazin matrices. This section is devoted to generalizing these notions to bounded finite potent endomorphisms of Hilbert spaces.

The Drazin-Star of a finite square complex matrix $A$ is $A^{D, *}=A^{D} A A^{*}$ and it is defined as the unique solution of the system of equations:

$$
\begin{aligned}
X\left(A^{\dagger}\right)^{*} X & =X \\
A^{r} X & =A^{r} A^{*} \\
X\left(A^{\dagger}\right)^{*} & =A^{D} A,
\end{aligned}
$$

where $i(A)=r$.

Analogously, the Star-Drazin of $A$ is the unique solution of

$$
\begin{aligned}
X\left(A^{\dagger}\right)^{*} X & =X \\
X A^{r} & =A^{*} A^{r} \\
\left(A^{\dagger}\right)^{*} X & =A^{D} A
\end{aligned}
$$

and its explicit expression is $A^{*, D}=A^{*} A A^{D}$.

\subsection{Drazin-Star Inverse of Bounded Finite Potent Endomorphisms}

We shall now extend the notion of the Drazin-Star matrix to bounded finite potent endomorphisms of arbitrary Hilbert spaces.

Definition 3.1. If $\mathcal{H}$ is a Hilbert space and $\varphi \in B_{f p}(\mathcal{H})$ with closed $\operatorname{Im} \varphi$ and $i(\varphi)=r$, we say that a linear map $\varphi^{D, *} \in \operatorname{End}_{\mathbb{C}}(\mathcal{H})$ is a Drazin-Star inverse of $\varphi$ when it satisfies that

- $\varphi^{D, *} \circ\left(\varphi^{\dagger}\right)^{*} \circ \varphi^{D, *}=\varphi^{D, *}$;

- $\varphi^{r} \circ \varphi^{D, *}=\varphi^{r} \circ \varphi^{*}$;

- $\varphi^{D, *} \circ\left(\varphi^{\dagger}\right)^{*}=\varphi^{D} \circ \varphi$. 
In the usual terminology of generalized matrices we have that $\varphi^{D \text {,* }}$ is a 2-inverse of $\left(\varphi^{\dagger}\right)^{*}$.

Lemma 3.2. If $\mathcal{H}$ be an arbitrary Hilbert space and $\varphi \in B_{f p}(\mathcal{H})$, then $\varphi^{D} \circ \varphi \circ \varphi^{*}$ is also a bounded finite potent endomorphism of $\mathcal{H}$.

Proof. If $\mathcal{H}=W_{\varphi} \oplus U_{\varphi}$ is the AST-decomposition of $\mathcal{H}$ induced by $\varphi$, one has that

$$
\operatorname{Im}\left(\varphi^{D} \circ \varphi \circ \varphi^{*}\right) \subseteq \operatorname{Im} \varphi^{D}=W_{\varphi}
$$

and, therefore, $\varphi^{D} \circ \varphi \circ \varphi^{*}$ is finite potent because $W_{\varphi}$ is a finite-dimensional C-vector space.

Hence, since $\varphi$ and $\varphi^{*}$ are bounded, and bearing in mind that from $[12$, Lemma 4.1] we know that $\varphi^{D}$ is bounded, we obtain that $\varphi^{D} \circ \varphi \circ \varphi^{*} \in B_{f p}(\mathcal{H})$.

Theorem 3.3. (Existence and uniqueness of the Drazin-Star inverse of a bounded finite potent endomorphism) Let $\mathcal{H}$ be a Hilbert space. Then, for every $\varphi \in B_{f p}(\mathcal{H})$ with closed $\operatorname{Im} \varphi$, there exists a unique Drazin-Star inverse $\varphi^{D, *} \in B_{f p}(\mathcal{H})$ with $\varphi^{D, *}=\varphi^{D} \circ \varphi \circ \varphi^{*}$.

Proof. Firstly, we shall check that $\varphi^{D} \circ \varphi \circ \varphi^{*}$ satisfies the conditions of the Drazin-Star inverse. Thus, given $\varphi \in B_{f p}(\mathcal{H})$ with closed $\operatorname{Im} \varphi$ and $i(\varphi)=r$, from the properties of the Drazin inverse and the Moore-Penrose inverse one has that

- $\varphi^{r} \circ\left(\varphi^{D} \circ \varphi \circ \varphi^{*}\right)=\left(\varphi^{r} \circ \varphi^{D} \circ \varphi\right) \circ \varphi^{*}=\varphi^{r} \circ \varphi^{*}$;

- $\left(\varphi^{D} \circ \varphi \circ \varphi^{*}\right) \circ\left(\varphi^{\dagger}\right)^{*}=\varphi^{D} \circ \varphi \circ\left(\varphi^{*} \circ\left(\varphi^{\dagger}\right)^{*}\right)=\varphi^{D} \circ\left(\varphi \circ \varphi^{\dagger} \circ \varphi\right)=\varphi^{D} \circ \varphi$;

- $\left(\varphi^{D} \circ \varphi \circ \varphi^{*}\right) \circ\left(\varphi^{\dagger}\right)^{*} \circ\left(\varphi^{D} \circ \varphi \circ \varphi^{*}\right)=\left(\varphi^{D} \circ \varphi \circ \varphi^{D}\right) \circ \varphi \circ \varphi^{*}=\varphi^{D} \circ \varphi \circ \varphi^{*}$.

Hence, $\varphi^{D} \circ \varphi \circ \varphi^{*}$ is a Drazin-Star inverse of $\varphi$.

Now, since $\varphi^{D} \circ \varphi=\left(\varphi^{D}\right)^{n} \circ \varphi^{n}$ for all $n \in \mathbb{N}$, for every Drazin-Star inverse $\varphi^{D, *} \in B_{f p}(\mathcal{H})$ we have that

$$
\begin{aligned}
\varphi^{D, *} & =\left(\varphi^{D, *} \circ\left(\varphi^{\dagger}\right)^{*}\right) \circ \varphi^{D, *}= \\
& =\left(\varphi^{D} \circ \varphi\right) \circ \varphi^{D, *}=\left(\varphi^{D}\right)^{r} \circ\left(\varphi^{r} \circ \varphi^{D, *}\right)= \\
& =\left(\varphi^{D}\right)^{r} \circ\left(\varphi^{r} \circ \varphi^{*}\right)=\varphi^{D} \circ \varphi \circ \varphi^{*},
\end{aligned}
$$

and the statement holds.

Corollary 3.4. If $\mathcal{H}$ is an arbitrary Hilbert space and $\varphi \in B_{\text {fp }}(\mathcal{H})$, then DrazinStar inverse $\varphi^{D, *}$ is admissible for the Moore-Penrose inverse.

Proof. The assertion is immediately deduced from Theorem 3.3 bearing in mind that $\operatorname{Im} \varphi^{D, *}$ is finite-dimensional and, therefore, closed. 


\subsection{Star-Drazin Inverse of Bounded Finite Potent Endomorphisms}

Our task is now to generalize to bounded finite potent endomorphisms of arbitrary Hilbert spaces the notion of the Star-Drazin matrix.

Definition 3.5. If $\mathcal{H}$ is a Hilbert space and $\varphi \in B_{f p}(\mathcal{H})$ with closed $\operatorname{Im} \varphi$ and $i(\varphi)=r$, we say that a linear map $\varphi^{*, D} \in \operatorname{End}_{\mathbb{C}}(\mathcal{H})$ is a Star-Drazin inverse of $\varphi$ when it satisfies that

- $\varphi^{*, D} \circ\left(\varphi^{\dagger}\right)^{*} \circ \varphi^{*, D}=\varphi^{*, D}$;

- $\varphi^{*, D} \circ \varphi^{r}=\varphi^{*} \circ \varphi^{r}$;

- $\left(\varphi^{\dagger}\right)^{*} \circ \varphi^{*, D}=\varphi \circ \varphi^{D}$.

Note that $\varphi^{*, D}$ is also a 2-inverse of $\left(\varphi^{\dagger}\right)^{*}$.

Lemma 3.6. If $\mathcal{H}$ is an arbitrary Hilbert space and $\varphi \in B_{f p}(\mathcal{H})$, then $\varphi^{*} \circ \varphi \circ \varphi^{D}$ is also a bounded finite potent endomorphism of $\mathcal{H}$.

Proof. If $\mathcal{H}=W_{\varphi} \oplus U_{\varphi}$ is again the AST-decomposition of $\mathcal{H}$ induced by $\varphi$, one has that

$$
\operatorname{Im}\left(\varphi^{*} \circ \varphi \circ \varphi^{D}\right)=\varphi^{*}\left(\varphi\left(W_{\varphi}\right)\right)
$$

and, therefore, $\varphi^{*} \circ \varphi \circ \varphi^{D}$ is finite potent.

Arguing now similarly to Lemma 3.2, we conclude that $\varphi^{*} \circ \varphi \circ \varphi^{D} \in$ $B_{f p}(\mathcal{H})$.

Theorem 3.7. (Existence and uniqueness of the Star-Drazin inverse of a bounded finite potent endomorphism) Let $\mathcal{H}$ be a Hilbert space. Then, for every $\varphi \in B_{f p}(\mathcal{H})$ with closed Im $\varphi$, there exists a unique Star-Drazin inverse $\varphi^{*, D} \in B_{f p}(\mathcal{H})$ with $\varphi^{*, D}=\varphi^{*} \circ \varphi \circ \varphi^{D}$.

Proof. Given $\varphi \in B_{f p}(\mathcal{H})$ with closed $\operatorname{Im} \varphi$ with $i(\varphi)=r$, one has that

- $\left(\varphi^{*} \circ \varphi \circ \varphi^{D}\right) \circ \varphi^{r}=\varphi^{*} \circ\left(\varphi \circ \varphi^{D} \circ \varphi^{r}\right)=\varphi^{*} \circ \varphi^{r}$;

- $\left.\left(\varphi^{\dagger}\right)^{*} \circ\left(\varphi^{*} \circ \varphi \circ \varphi^{D}\right)=\left(\left(\varphi^{\dagger}\right)^{*} \circ \varphi^{*}\right) \circ \varphi \circ \varphi^{D}\right)=\left(\varphi \circ \varphi^{\dagger} \circ \varphi\right) \circ \varphi^{D}=\varphi \circ \varphi^{D}$;

- $\left(\varphi^{*} \circ \varphi \circ \varphi^{D}\right) \circ\left(\varphi^{\dagger}\right)^{*} \circ\left(\varphi^{*} \circ \varphi \circ \varphi^{D}\right)=\left(\varphi^{*} \circ \varphi \circ \varphi^{D}\right) \circ \varphi \circ \varphi^{D}=\varphi^{*} \circ \varphi \circ \varphi^{D}$; from where we deduce that $\varphi^{*} \circ \varphi \circ \varphi^{D}$ is a Star-Drazin inverse of $\varphi$. Finally, for every Star-Drazin inverse $\varphi^{*, D} \in B_{f p}(\mathcal{H})$ we have that

$$
\begin{aligned}
\varphi^{*, D} & =\varphi^{*, D} \circ\left(\left(\varphi^{\dagger}\right)^{*} \circ \varphi^{*, D}\right)= \\
& =\varphi^{*, D} \circ\left(\varphi^{D} \circ \varphi\right)=\left(\varphi^{*, D} \circ \varphi^{r}\right) \circ\left(\varphi^{D}\right)^{r}= \\
& =\left(\varphi^{*} \circ \varphi^{r}\right) \circ\left(\varphi^{D}\right)^{r}=\varphi^{*} \circ \varphi \circ \varphi^{D},
\end{aligned}
$$

and the claim is proved.

A direct consequence of Theorem 3.7 is:

Corollary 3.8. If $\mathcal{H}$ is an arbitrary Hilbert space and $\varphi \in B_{\text {fp }}(\mathcal{H})$ with closed $\operatorname{Im} \varphi$, then Star-Drazin inverse $\varphi^{*, D}$ is admissible for the Moore-Penrose inverse. 
Proposition 3.9. If $\mathcal{H}$ is an arbitrary Hilbert space and $\varphi \in B_{\text {fp }}(\mathcal{H})$ with closed $\operatorname{Im} \varphi$, then $\left(\varphi^{*}\right)^{*, D}=\left(\varphi^{D, *}\right)^{*}$ and $\left(\varphi^{*}\right)^{D, *}=\left(\varphi^{*, D}\right)^{*}$.

Proof. From the explicit expressions of $\varphi^{D, *}$ and $\varphi^{*, D}$ obtained in Theorem 3.3 and Theorem 3.7, bearing in mind that $\left(\varphi^{*}\right)^{D}=\left(\varphi^{D}\right)^{*}$ one has that

$$
\left(\varphi^{*}\right)^{*, D}=\left(\varphi^{*}\right)^{*} \circ \varphi^{*} \circ\left(\varphi^{*}\right)^{D}=\left(\varphi^{D} \circ \varphi \circ \varphi^{*}\right)^{*}=\left(\varphi^{D, *}\right)^{*}
$$

and, similarly, one can easily check that $\left(\varphi^{*}\right)^{D, *}=\left(\varphi^{*, D}\right)^{*}$.

Accordingly, the claim is proved.

\subsection{Properties of the Drazin-Star and the Star-Drazin Inverses}

We shall now study the main properties of the Drazin-Star and the Star-Drazin inverses of a bounded finite potent endomorphism $\varphi \in B_{f p}(\mathcal{H})$.

Lemma 3.10. Given a Hilbert space $\mathcal{H}$ and a linear map $\varphi \in B_{\text {fp }}(\mathcal{H})$ with closed $\operatorname{Im} \varphi$, then $\varphi^{\dagger} \circ \varphi \circ\left(\varphi^{*}\right)^{D}=\left(\varphi^{*}\right)^{D}$.

Proof. According to Proposition 2.3, we know that $\varphi^{*} \in B_{f p}(\mathcal{H})$ and let us consider the AST-decomposition $\mathcal{H}=W_{\varphi^{*}} \oplus U_{\varphi^{*}}$ determined by $\varphi^{*}$.

If $\mathcal{H}=W_{\varphi} \oplus U_{\varphi}$ is the AST-decomposition induced by $\varphi$, it follows from $[14$, Proposition 4.5] that

$$
\operatorname{Im}\left(\varphi^{*}\right)^{D}=W_{\varphi^{*}}=U_{\varphi}^{\perp} \subseteq[\operatorname{Ker} f]^{\perp},
$$

and the statement is immediately deduced from Lemma 2.6.

Corollary 3.11. If $\mathcal{H}$ is a Hilbert space and $\varphi \in B_{\text {fp }}(\mathcal{H})$ with closed Im $\varphi$, then one has that

(1) $\varphi^{D} \circ \varphi^{\dagger} \circ \varphi=\varphi^{D}$;

(2) $\varphi^{D} \circ \varphi^{*} \circ\left(\varphi^{\dagger}\right)^{*}=\varphi^{D}$.

Proof. Both equalities are direct consequence of Lemma 3.10.

Proposition 3.12. If $\mathcal{H}$ is a Hilbert space and $\varphi \in B_{\text {fp }}(\mathcal{H})$ with closed $\operatorname{Im} \varphi$, then the following conditions are equivalent:

(1) $\left(\varphi^{\dagger}\right)^{*} \circ \varphi^{D, *} \circ\left(\varphi^{\dagger}\right)^{*}=\left(\varphi^{\dagger}\right)^{*}$;

(2) $\varphi^{\dagger} \circ \varphi \circ \varphi^{D} \circ \varphi=\varphi^{\dagger} \circ \varphi$;

(3) $\varphi \circ \varphi^{D} \circ \varphi=\varphi$;

(4) $\varphi \circ \varphi^{D} \circ \varphi \circ \varphi^{\dagger}=\varphi \circ \varphi^{\dagger}$;

(5) $\left(\varphi^{\dagger}\right)^{*} \circ \varphi^{*, D} \circ\left(\varphi^{\dagger}\right)^{*}=\left(\varphi^{\dagger}\right)^{*}$.

Proof. 1) $\Longleftrightarrow 2$ ) If $\left(\varphi^{\dagger}\right)^{*} \circ \varphi^{D, *} \circ\left(\varphi^{\dagger}\right)^{*}=\left(\varphi^{\dagger}\right)^{*}$, then

$$
\varphi^{*} \circ\left(\varphi^{\dagger}\right)^{*} \circ \varphi^{D, *} \circ\left(\varphi^{\dagger}\right)^{*}=\varphi^{*} \circ\left(\varphi^{\dagger}\right)^{*}
$$

and one has that

$$
\varphi^{\dagger} \circ \varphi \circ \varphi^{D} \circ \varphi=\left(\varphi^{\dagger} \circ \varphi\right)^{*} \circ \varphi^{D} \circ \varphi \circ\left(\varphi^{\dagger} \circ \varphi\right)^{*}=\left(\varphi^{\dagger} \circ \varphi\right)^{*}=\varphi^{\dagger} \circ \varphi \text {. }
$$


Conversely, bearing in mind that $\varphi^{\dagger} \circ \varphi \circ \varphi^{D} \circ \varphi=\varphi^{\dagger} \circ \varphi$ is equivalent to

$$
\varphi^{*} \circ\left(\varphi^{\dagger}\right)^{*} \circ \varphi^{D, *} \circ\left(\varphi^{\dagger}\right)^{*}=\varphi^{*} \circ\left(\varphi^{\dagger}\right)^{*},
$$

in this case, from Proposition 2.7, we obtain that

$$
\begin{aligned}
\left(\varphi^{\dagger}\right)^{*} \circ \varphi^{D, *} \circ\left(\varphi^{\dagger}\right)^{*}=\left(\left(\varphi^{\dagger}\right)^{*} \circ \varphi^{*} \circ\left(\varphi^{\dagger}\right)^{*}\right) \circ \varphi^{D, *} \circ\left(\varphi^{\dagger}\right)^{*}=\left(\varphi^{\dagger}\right)^{*} \circ \varphi^{*} \circ\left(\varphi^{\dagger}\right)^{*}=\left(\varphi^{\dagger}\right)^{*} . & \\
(2) \Longleftrightarrow & (3) \text { When } \varphi^{\dagger} \circ \varphi \circ \varphi^{D} \circ \varphi=\varphi^{\dagger} \circ \varphi, \text { one has that } \\
& \varphi \circ \varphi^{D} \circ \varphi=\left(\varphi \circ \varphi^{\dagger} \circ \varphi\right) \circ \varphi^{D} \circ \varphi=\varphi \circ \varphi^{\dagger} \circ \varphi=\varphi,
\end{aligned}
$$

from where the assertion is proved.

$(3) \Longleftrightarrow(4)$ Assuming that $\varphi \circ \varphi^{D} \circ \varphi=\varphi$, we immediately check that

$$
\varphi \circ \varphi^{D} \circ \varphi \circ \varphi^{\dagger}=\varphi \circ \varphi^{\dagger}
$$

and the converse implication is deduced from the equality $\varphi \circ \varphi^{\dagger} \circ \varphi=\varphi$.

$(4) \Longleftrightarrow(5)$ Since $\left(\varphi^{\dagger}\right)^{*} \circ \varphi^{*, D}=\varphi \circ \varphi^{D}$, if $\varphi \circ \varphi^{D} \circ \varphi \circ \varphi^{\dagger}=\varphi \circ \varphi^{\dagger}$ one has that

$$
\left(\varphi^{\dagger}\right)^{*} \circ \varphi^{*, D} \circ\left(\varphi \circ \varphi^{\dagger}\right)^{*}=\left(\varphi \circ \varphi^{\dagger}\right)^{*},
$$

and it follows from Proposition 2.7 that

$$
\left(\varphi^{\dagger}\right)^{*} \circ \varphi^{*, D} \circ\left(\varphi^{\dagger}\right)^{*}=\left(\varphi^{\dagger}\right)^{*} \circ \varphi^{*, D} \circ\left(\varphi \circ \varphi^{\dagger}\right)^{*} \circ\left(\varphi^{\dagger}\right)^{*}=\left(\varphi \circ \varphi^{\dagger}\right)^{*} \circ\left(\varphi^{\dagger}\right)^{*}=\left(\varphi^{\dagger}\right)^{*} \text {. }
$$

Conversely, if $\left(\varphi^{\dagger}\right)^{*} \circ \varphi^{*, D} \circ\left(\varphi^{\dagger}\right)^{*}=\left(\varphi^{\dagger}\right)^{*}$, we have that

$$
\varphi \circ \varphi^{D} \circ\left(\varphi^{\dagger}\right)^{*} \circ \varphi^{*}=\left(\varphi^{\dagger}\right)^{*} \circ \varphi^{*}
$$

and we obtain that $\varphi \circ \varphi^{D} \circ \varphi \circ \varphi^{\dagger}=\varphi \circ \varphi^{\dagger}$.

With the terminology of generalized inverses, conditions of Proposition 3.12 determine that $\varphi^{D, *}$ and $\varphi^{*, D}$ are reflexive generalized inverses of $\left(\varphi^{\dagger}\right)^{*}$.

Lemma 3.13. If $\mathcal{H}$ is a Hilbert space and $\varphi \in B_{f p}(\mathcal{H})$ with closed Im $\varphi$, then the conditions of Proposition 3.12 hold if and only if $i(\varphi) \leq 1$.

Proof. Since $\varphi \circ \varphi^{D} \circ \varphi=\varphi$ and the conditions of Proposition 3.12 hold if and only if $\varphi \circ \varphi^{D} \circ \varphi=\varphi$, the claim is deduced bearing in mind that " $i(\varphi) \leq 1$ if and only if $\varphi=\varphi_{1}$ ".

A direct consequence of Lemma 3.13 is

Lemma 3.14. If $\mathcal{H}$ is a Hilbert space and $\varphi \in B_{\text {fp }}(\mathcal{H})$ with closed Im $\varphi$, then $\varphi^{D, *}$ and $\varphi^{*, D}$ are reflexive generalized inverses of $\left(\varphi^{\dagger}\right)^{*}$ if and only if $i(\varphi) \leq 1$.

Proposition 3.15. Given a Hilbert space $\mathcal{H}$, if $\varphi \in B_{\text {fp }}(\mathcal{H})$ with closed $\operatorname{Im} \varphi$ with $i(\varphi)=r)$, then the following equalities are equivalent:

(1) $\varphi^{r} \circ \varphi^{D, *} \circ \varphi^{r}=\varphi^{r}$;

(2) $\varphi^{r} \circ \varphi^{*} \circ \varphi^{r}=\varphi^{r}$;

(3) $\varphi^{r} \circ \varphi^{*, D} \circ \varphi^{r}=\varphi^{r}$. 
Proof. Bearing in mind that $\varphi^{r} \circ \varphi^{D, *}=\varphi^{r} \circ \varphi^{*}$ and $\varphi^{*, D} \circ \varphi^{r}=\varphi^{*} \circ \varphi^{r}$, one immediately obtains that 1) $\Longleftrightarrow 2$ ) and 2)

Lemma 3.16. If $\mathcal{H}$ is a Hilbert space and $\varphi \in B_{\text {fp }}(\mathcal{H})$ with closed Im $\varphi$, then

$$
\varphi^{\dagger} \circ \varphi^{D, *}=\varphi^{\dagger} \circ \varphi^{D} \text { if and only if } \varphi^{D, *}=\varphi^{D} \text {. }
$$

Proof. Since $\operatorname{Im} \varphi^{D, *} \subset \operatorname{Im} \varphi$ and $\operatorname{Im} \varphi^{D} \subseteq \operatorname{Im} \varphi$, from Lemma 2.5 we have that $\varphi \circ \varphi^{\dagger} \circ \varphi^{D, *}=\varphi^{D, *}$ and $\varphi \circ \varphi^{\dagger} \circ \varphi^{D}=\varphi^{D}$. Accordingly, from these equalities the statement is immediately deduced.

Lemma 3.17. Given a Hilbert space $\mathcal{H}$ and an endomorphism $\varphi \in B_{f p}(\mathcal{H})$ with closed $\operatorname{Im} \varphi$, then

$$
\varphi^{*, D} \circ \varphi^{\dagger}=\varphi^{D} \circ \varphi^{\dagger} \text { if and only if } \varphi^{*, D}=\varphi^{D} .
$$

Proof. The claim is immediately deduced from Corollary 3.11.

Lemma 3.18. If $\mathcal{H}$ is a Hilbert space, for every $\varphi \in B_{\text {fp }}(\mathcal{H})$ with closed $\operatorname{Im} \varphi$, one has that

$$
\varphi \circ \varphi^{D, *}=\varphi \circ \varphi^{D} \text { if and only if } \varphi^{D, *}=\varphi^{D}
$$

and

$$
\varphi^{*, D} \circ \varphi=\varphi \circ \varphi^{D} \text { if and only if } \varphi^{*, D}=\varphi^{D} .
$$

Proof. The assertions are direct consequence of the equalities $\varphi^{D} \circ \varphi=\varphi \circ \varphi^{D}$ and $\varphi^{D} \circ \varphi \circ \varphi^{D}=\varphi^{D}$, which are well-known properties of the Drazin inverse of a finite potent endomorphisms.

Now, it follows from Lemmas 3.16, 3.17 and 3.18 that

Corollary 3.19. Given a Hilbert space $\mathcal{H}$ and an endomorphism $\varphi \in B_{\text {fp }}(\mathcal{H})$ with closed $\operatorname{Im} \varphi$, then we have that

$$
\varphi^{\dagger} \circ \varphi^{D, *}=\varphi^{\dagger} \circ \varphi^{D} \text { if and only if } \varphi \circ \varphi^{D, *}=\varphi \circ \varphi^{D}
$$

and

$$
\varphi^{*, D} \circ \varphi^{\dagger}=\varphi^{D} \circ \varphi^{\dagger} \text { if and only if } \varphi^{*, D} \circ \varphi=\varphi \circ \varphi^{D} \text {. }
$$

Lemma 3.20. Given a Hilbert space $\mathcal{H}$ and a endomorphism $\varphi \in B_{\text {fp }}(\mathcal{H})$ with closed $\operatorname{Im} \varphi$, then

$$
\varphi^{D, *} \circ \varphi=\varphi \circ \varphi^{D} \text { if and only if } \varphi^{D, *}=\varphi^{d, \dagger} \text {. }
$$

Proof. Bearing in mind that from Lemma 2.8 we know that $\varphi^{*} \circ \varphi \circ \varphi^{\dagger}=\varphi^{*}$, if $\varphi^{D, *} \circ \varphi=\varphi \circ \varphi^{D}$ one immediately deduces that

$$
\varphi^{D, *}=\varphi^{D} \circ \varphi \circ\left(\varphi^{*} \circ \varphi \circ \varphi^{\dagger}\right)=\varphi^{D} \circ \varphi \circ \varphi^{\dagger}=\varphi^{d, \dagger} .
$$

Conversely, when $\varphi^{D, *}=\varphi^{d, \dagger}$, we have that

$$
\varphi^{D, *} \circ \varphi=\varphi^{D} \circ \varphi \circ \varphi^{\dagger} \circ \varphi=\varphi^{D} \circ \varphi,
$$

and the claim is proved. 
Lemma 3.21. If $\mathcal{H}$ is a Hilbert space and $\varphi \in B_{\text {fp }}(\mathcal{H})$ with closed Im $\varphi$, then one has that

(1) $\varphi^{D, *} \circ \varphi=\varphi^{\dagger} \circ \varphi$ if and only if $\varphi^{D, *}=\varphi^{\dagger}$;

(2) $\varphi \circ \varphi^{D, *}=\varphi \circ \varphi^{\dagger}$ if and only if $\varphi \circ \varphi^{D, *} \circ \varphi=\varphi$.

Proof. Similar to the proof of Lemma 3.20, both assertions can easily be deduced from the equality $\varphi^{*} \circ \varphi \circ \varphi^{\dagger}=\varphi^{*}$.

Lemma 3.22. Given a Hilbert space $\mathcal{H}$ and a finite potent endomorphism $\varphi \in$ $B_{f p}(\mathcal{H})$ with closed $\operatorname{Im} \varphi$, then one has that $\varphi^{d, \dagger}=\varphi^{D, *} \circ\left(\varphi^{\dagger}\right)^{*} \circ \varphi^{\dagger}$ and $\varphi^{\dagger, d}=\varphi^{\dagger} \circ\left(\varphi^{\dagger}\right)^{*} \circ \varphi^{*, D}$.

Proof. From the explicit expressions of $\varphi^{d, \dagger}, \varphi^{\dagger, d}, \varphi^{D, *}$ and $\varphi^{*, D}$ and from the properties of the Moore-Penrose inverse, an easy computation shows that the statement holds.

Lemma 3.23. For every bounded finite potent endomorphism $\varphi \in B_{f p}(\mathcal{H})$ with $\mathcal{H}$ being an arbitrary Hilbert space, one has that

$$
\varphi^{D, *}=\varphi^{*} \text { if and only if } \varphi^{d, \dagger}=\varphi^{\dagger} .
$$

Proof. Let us assume that $\varphi^{D, *}=\varphi^{*}$. With this hypothesis, it follows from Lemma 3.22 that

$$
\varphi^{d, \dagger}=\varphi^{D, *} \circ\left(\varphi^{\dagger}\right)^{*} \circ \varphi^{\dagger}=\varphi^{*} \circ\left(\varphi^{\dagger}\right)^{*} \circ \varphi^{\dagger}=\varphi^{\dagger} .
$$

Conversely, if $\varphi^{d, \dagger}=\varphi^{\dagger}$, it follows from Lemma 2.8 that $\varphi^{\dagger} \circ \varphi \circ \varphi^{*}=\varphi^{*}$ and one deduces that

$$
\varphi^{D, *}=\varphi^{D} \circ \varphi \circ\left(\varphi^{\dagger} \circ \varphi \circ \varphi^{*}\right)=\varphi^{\dagger} \circ \varphi \circ \varphi^{*}=\varphi^{*} .
$$

Lemma 3.24. Given a Hilbert space $\mathcal{H}$ and a finite potent endomorphism $\varphi \in$ $B_{f p}(\mathcal{H})$ with closed $\operatorname{Im} \varphi$, we have that

(1) $\varphi \circ \varphi^{*, D}=\varphi \circ \varphi^{D}$ if and only if $\varphi^{*, D}=\varphi^{\dagger, d}$;

(2) $\varphi \circ \varphi^{*, D}=\varphi \circ \varphi^{\dagger}$ if and only if $\varphi^{*, D}=\varphi^{\dagger}$;

(3) $\varphi^{*, D} \circ \varphi=\varphi^{\dagger} \circ \varphi$ if and only if $\varphi \circ \varphi^{*, D} \circ \varphi=\varphi$.

Proof. These statements hold again from the equality $\varphi^{\dagger} \circ \varphi \circ \varphi^{*}=\varphi^{*}$.

Lemma 3.25. If $\mathcal{H}$ is a Hilbert space and $\varphi \in B_{\text {fp }}(\mathcal{H})$ with closed $\operatorname{Im} \varphi$, then $\varphi^{*, D}=\varphi^{*}$ if and only if $\varphi^{\dagger, d}=\varphi^{\dagger}$.

Proof. Let us assume that $\varphi^{*, D}=\varphi^{*}$. In this case, one has that

$$
\varphi \circ \varphi^{D}=\left(\varphi^{\dagger}\right)^{*} \circ\left(\varphi^{*} \circ \varphi \circ \varphi^{D}\right)=\left(\varphi^{\dagger}\right)^{*} \circ \varphi^{*}=\varphi \circ \varphi^{\dagger}
$$

and, therefore, we deduce that

$$
\varphi^{\dagger, d}=\varphi^{\dagger} \circ \varphi \circ \varphi^{D}=\varphi^{\dagger} \circ \varphi \circ \varphi^{\dagger}=\varphi^{\dagger} .
$$


Conversely, if $\varphi^{\dagger, d}=\varphi^{\dagger}$, bearing in mind again that $\varphi^{*} \circ \varphi \circ \varphi^{\dagger}=\varphi^{*}$, one has that

$$
\varphi^{*, D}=\varphi^{*} \circ \varphi \circ \varphi^{\dagger, d}=\varphi^{*} \circ \varphi \circ \varphi^{\dagger}=\varphi^{*}
$$

from where the claim is deduced.

We shall now illustrate the previous results with the following example.

Example 3.26. Let $\left\{u_{i}\right\}_{i \in \mathbb{N}}$ be an orthonormal basis of a separable Hilbert space $\mathcal{H}$. If we consider $\varphi \in B_{f p}(\mathcal{H})$ determined by the conditions

$$
\varphi\left(u_{i}\right)= \begin{cases}u_{1}+u_{2}+u_{4} & \text { if } i=1 \\ 2 u_{1}+u_{3} & \text { if } i=2 \\ u_{1}-2 u_{2}+3 u_{3}-2 u_{4} & \text { if } i=3 \\ 0 & \text { if } i=4 \\ \frac{1}{i^{2}} u_{4} & \text { if } i \geq 5\end{cases}
$$

an easy computation shows that

$$
\varphi^{*}\left(u_{i}\right)=\left\{\begin{array}{ll}
u_{1}+2 u_{2}+u_{3} & \text { if } i=1 \\
u_{1}-2 u_{3} & \text { if } i=2 \\
u_{2}+3 u_{3} & \text { if } i=3 \\
u_{1}-2 u_{3}+\sum_{j \geq 5} \frac{1}{j^{2}} u_{j} & \text { if } i=4 \\
0 & \text { if } i \geq 5
\end{array} .\right.
$$

Thus, since $W_{\varphi}=\left\langle u_{1}, u_{2}+u_{4}, u_{3}\right\rangle$ and $U_{\varphi}=\overline{\left\langle u_{i}\right\rangle_{i \geq 4}}$, one has that

- $W_{\varphi^{*}}=U_{\varphi}^{\perp}=\left\langle u_{1}, u_{2}, u_{3}\right\rangle$;

- $U_{\varphi^{*}}=W_{\varphi}^{\perp}=\left\langle u_{2}-u_{4}\right\rangle \oplus \overline{\left\langle u_{j}\right\rangle_{j \geq 5}}$.

Also, it is clear that $i(\varphi)=i\left(\varphi^{*}\right)=2$.

Now, from the explicit expression of the Drazin inverse

$$
\varphi^{D}\left(u_{i}\right)=\left\{\begin{array}{ll}
-\frac{2}{3} u_{1}+u_{2}-\frac{1}{3} u_{3}+u_{4} & \text { if } i=1 \\
\frac{5}{3} u_{1}-u_{2}+\frac{1}{3} u_{3}-u_{4} & \text { if } i=2 \\
\frac{4}{3} u_{1}-u_{2}+\frac{2}{3} u_{3}-u_{4} & \text { if } i=3 \\
0 & \text { if } i \geq 4
\end{array},\right.
$$

a non-difficult calculation shows that

$$
\varphi^{D, *}\left(u_{i}\right)= \begin{cases}u_{1}+2 u_{2}+u_{3}+2 u_{4} & \text { if } i=1 \\ u_{1}-2 u_{3} & \text { if } i=2 \\ u_{2}+3 u_{3}+u_{4} & \text { if } i=3 \\ u_{1}-2 u_{3} & \text { if } i=4 \\ 0 & \text { if } i \geq 5\end{cases}
$$


and

$$
\varphi^{*, D}\left(u_{i}\right)=\left\{\begin{array}{ll}
u_{1}+2 u_{2}+u_{3} & \text { if } i=1 \\
2 u_{1}-4 u_{3}+\sum_{j \geq 5} \frac{1}{j^{2}} u_{j} & \text { if } i=2 \\
u_{2}+3 u_{3} & \text { if } i=3 \\
0 & \text { if } i \geq 4
\end{array} .\right.
$$

Moreover, since one can obtain thar $\varphi^{\dagger}$ is determined by the assignations

$$
\varphi^{\dagger}\left(u_{i}\right)= \begin{cases}-\frac{2}{3} u_{1}+u_{2}-\frac{1}{3} u_{3} & \text { if } i=1 \\ \frac{5}{3} u_{1}-u_{2}+\frac{1}{3} u_{3}-\frac{1}{\lambda} \sum_{j \geq 5}\left(\frac{1}{j^{2}} u_{j}\right) & \text { if } i=2 \\ \frac{4}{3} u_{1}-u_{2}+\frac{2}{3} u_{3} & \text { if } i=3 \\ \frac{1}{\lambda} \sum_{j \geq 5}\left(\frac{1}{j^{2}} u_{j}\right) & \text { if } i=4 \\ 0 & \text { if } i \geq 5\end{cases}
$$

with $\lambda=\sum_{j \geq 5} \frac{1}{j^{4}}=\frac{\pi^{4}}{90}-\frac{827}{768}$, then

$$
\left(\varphi^{*}\right)^{\dagger}\left(u_{i}\right)=\left(\varphi^{\dagger}\right)^{*}\left(u_{i}\right)=\left\{\begin{array}{ll}
-\frac{2}{3} u_{1}+\frac{5}{3} u_{2}+\frac{4}{3} u_{3} & \text { if } i=1 \\
u_{1}-u_{2}-u_{3} & \text { if } i=2 \\
-\frac{1}{3} u_{1}+\frac{1}{3} u_{2}+\frac{2}{3} u_{3} & \text { if } i=3 \\
0 & \text { if } i=4 \\
\frac{-1}{\lambda j^{2}}\left(u_{2}-u_{4}\right) & \text { if } i \geq 5
\end{array},\right.
$$

and readers can easily check that $\varphi^{D, *}$ and $\varphi^{*, D}$ satisfy the conditions of Definition 3.1 and Definition 3.5 respectively.

Remark 3.2\%. Given a complex finite matrix $A$, from the above properties of $\varphi^{D, *}$ and $\varphi^{*, D}$ we recover all the statements offered in [10, Lemma 2.2] and we obtain the following new properties:

- $A^{D, *}$ and $A^{*, D}$ are reflexive generalized inverses of $\left(A^{\dagger}\right)^{*}$ if and only if $i(A) \leq 1$

- if $A^{c \dagger}$ is the CMP inverse of $A$, then $A^{D, *}$ and $A^{*, D}$ are reflexive generalized inverses of $\left(A^{c \dagger}\right)^{*}$ if and only if $i(A) \leq 1$;

- $A^{\dagger} A^{D, *}=A^{\dagger} A^{D}$ if and only if $A^{D, *}=A^{D}$;

- $A^{*, D} A^{\dagger}=A^{D} A^{\dagger}$ if and only if $A^{*, D}=A^{D}$;

- $A^{d, \dagger}=A^{D, *}\left(A^{\dagger}\right)^{*} A^{\dagger}$;

- $A^{\dagger, d}=A^{\dagger}\left(A^{\dagger}\right)^{*} A^{*, D}$.

\subsection{Group-Star and Star-Group of Bounded Finite Potent Endomorphisms}

Let $\mathcal{H}$ be again a Hilbert space. We shall now extend the notions of Group-Star and Star-Group matrices to bounded finite potent endomorphism of $\mathcal{H}$ that has been offered in [10, Section 3]. 
If $\varphi \in B_{f p}(\mathcal{H}), \mathcal{H}=W_{\varphi} \oplus U_{\varphi}$ is the AST-decomposition induced by $\varphi$ and $i(\varphi) \leq 1$, then we have that $\varphi$ is admissible for the Moore-Penrose inverse because $\operatorname{Im} \varphi=W_{\varphi}$, which is a finite-dimensional vector space.

Definition 3.28. If $\mathcal{H}$ is a Hilbert space and $\varphi \in B_{f p}(\mathcal{H})$ with $i(\varphi) \leq 1$, we say that a linear map $\varphi^{\#, *} \in \operatorname{End}_{\mathbb{C}}(\mathcal{H})$ is a Group-Star inverse of $\varphi$ when it satisfies that

- $\varphi^{\#, *} \circ\left(\varphi^{\dagger}\right)^{*} \circ \varphi^{\#, *}=\varphi^{\#, *}$;

- $\varphi \circ \varphi^{\#, *}=\varphi \circ \varphi^{*}$;

- $\varphi^{\#, *} \circ\left(\varphi^{\dagger}\right)^{*}=\varphi^{\#} \circ \varphi$.

The existence and uniqueness of the Group-Star inverse of a bounded finite potent endomorphism $\varphi \in B_{f p}(\mathcal{H})$ with $i(\varphi) \leq 1$ is immediately deduced from Theorem 3.3. We have that $\varphi^{\#, *} \in B_{f p}(\mathcal{H})$ and its explicit expression is $\varphi^{\#, *}=\varphi^{\#} \circ \varphi \circ \varphi^{*}$.

Remark 3.29. In [10] the existence and uniqueness of $A^{\#, *}$ is proved for matrices $A \in \operatorname{Mat}_{n \times n}(\mathbb{C})$ with $i(A)=1$. Bearing in mind that the index of $\varphi \in B_{f p}(\mathcal{H})$ is zero if and only if $\mathcal{H}$ is finite-dimensional and $\varphi \in \operatorname{Aut}_{\mathbb{C}}(\mathcal{H})$, it is clear that for $\varphi^{\#, *}=\varphi^{*}$ all the conditions of Definition 3.28 hold. Accordingly, if $A \in \operatorname{Mat}_{n \times n}(\mathbb{C})$ with $i(A)=0$, one has that $A^{\#, *}=A^{*}$ satisfies the equations of $[10$, Corollary 3.1.a)] from where the Group-Star matrix is defined.

Definition 3.30. If $\mathcal{H}$ is a Hilbert space and $\varphi \in B_{f p}(\mathcal{H})$ with $i(\varphi) \leq 1$, we say that a linear map $\varphi^{*, \#} \in \operatorname{End}_{\mathbb{C}}(\mathcal{H})$ is a Star-Group inverse of $\varphi$ when it satisfies that

- $\varphi^{*, \#} \circ\left(\varphi^{\dagger}\right)^{*} \circ \varphi^{*, \#}=\varphi^{*, \#}$

- $\varphi^{*, \#} \circ \varphi=\varphi^{*} \circ \varphi$;

- $\left(\varphi^{\dagger}\right)^{*} \circ \varphi^{*, \#}=\varphi^{\#} \circ \varphi$.

For every $\varphi \in B_{f p}(\mathcal{H})$ with $i(\varphi) \leq 1$, Theorem 3.7 shows that $\varphi^{*}$,\# exists and is unique, $\varphi^{*, \#} \in B_{f p}(\mathcal{H})$ and $\varphi^{*, \#}=\varphi^{*} \circ \varphi \circ \varphi^{\#}$.

Moreover, similar to above, when $i(\varphi)=0$ one has that $\varphi^{*, \#}=\varphi^{*}$ and if $A \in \operatorname{Mat}_{n \times n}(\mathbb{C})$ with $i(A)=0$, then $A^{\#, *}=A^{*}$.

Furthermore, if $\varphi \in B_{f p}(\mathcal{H})$ is the same as in this section, it follows from Proposition 3.9 that $\left(\varphi^{*, \#}\right)^{*}=\left(\varphi^{*}\right)^{\#, *}$ and $\left(\varphi^{\#, *}\right)^{*}=\left(\varphi^{*}\right)^{*, \#}$.

If $\mathcal{H}$ is a Hilbert space and $\varphi \in B_{f p}(\mathcal{H})$ with $i(\varphi) \leq 1$, from Proposition 3.12 and Lemma 3.13 we deduce that $\varphi^{\#, *}$ and $\varphi^{*, \#}$ are reflexive inverses of $\left(\varphi^{\dagger}\right)^{*}$. Indeed, one has that

$$
\left(\varphi^{\dagger}\right)^{*} \circ \varphi^{\#, *} \circ\left(\varphi^{\dagger}\right)^{*}=\left(\varphi^{\dagger}\right)^{*}
$$

and

$$
\left(\varphi^{\dagger}\right)^{*} \circ \varphi^{*, \#} \circ\left(\varphi^{\dagger}\right)^{*}=\left(\varphi^{\dagger}\right)^{*}
$$


Proposition 3.31. Given a Hilbert space $\mathcal{H}$ and $\varphi \in B_{\text {fp }}(\mathcal{H})$ with $i(\varphi) \leq 1$, then the following equalities are equivalent:

(1) $\varphi \circ \varphi^{\#, *} \circ \varphi=\varphi$;

(2) $\varphi \circ \varphi^{*} \circ \varphi=\varphi$;

(3) $\varphi \circ \varphi^{*, \#} \circ \varphi=\varphi$;

(4) $\varphi \circ \varphi^{\#, *}=\varphi \circ \varphi^{\dagger}$;

(5) $\varphi^{*, \#} \circ \varphi=\varphi^{\dagger} \circ \varphi$.

Proof. Bearing in mind that from Definitions 3.28 and 3.30 we know that $\varphi \circ \varphi^{\#, *}=\varphi \circ \varphi^{*}$ and $\varphi^{*, \#} \circ \varphi=\varphi^{*} \circ \varphi$, it is clear that $(1) \Longleftrightarrow(2) \Longleftrightarrow(3)$.

$(1) \Longleftrightarrow$ (4) If $\varphi \circ \varphi^{\#, *} \circ \varphi=\varphi$, it follows from Lemma 2.8 that $\varphi^{*} \circ \varphi \circ \varphi^{\dagger}=$ $\varphi^{*}$ and, therefore, one obtains that

$$
\varphi \circ \varphi^{\#, *}=\varphi \circ \varphi^{\#, *} \circ \varphi \circ \varphi^{\dagger}=\varphi \circ \varphi^{\dagger} .
$$

Conversely, when $\varphi \circ \varphi^{\#, *}=\varphi \circ \varphi^{\dagger}$, one has that

$$
\varphi \circ \varphi^{\#, *} \circ \varphi=\varphi \circ \varphi^{\dagger} \circ \varphi=\varphi,
$$

from where this equivalence is deduced.

$(3) \Longleftrightarrow(5)$ Let us assume that $\varphi \circ \varphi^{*}, \# \circ \varphi=\varphi$. In this case, bearing in mind that Lemma 2.8 also shows that $\varphi^{\dagger} \circ \varphi \circ \varphi^{*}=\varphi^{*}$, one has that

$$
\varphi^{*, \#} \circ \varphi=\varphi^{\dagger} \circ \varphi \circ \varphi^{*, \#} \circ \varphi=\varphi^{\dagger} \circ \varphi .
$$

On the other hand, if $\varphi^{*}, \# \circ \varphi=\varphi^{\dagger} \circ \varphi$, then one has that

$$
\varphi \circ \varphi^{*, \#} \circ \varphi=\varphi
$$

and the statement is proved.

Lemma 3.32. If $\mathcal{H}$ is a Hilbert space and $\varphi \in B_{\text {fp }}(\mathcal{H})$ with $i(\varphi) \leq 1$, one has that

(1) $\varphi^{\#, *} \circ \varphi=\varphi^{\dagger} \circ \varphi$ if and only if $\varphi^{\#, *}=\varphi^{\dagger}$;

(2) $\varphi \circ \varphi^{*, \#}=\varphi \circ \varphi^{\dagger}$ if and only if $\varphi^{*, \#}=\varphi^{\dagger}$.

Proof. (1) This assertion follows from Lemma 3.21 (1).

(2) This claim is immediately deduced from Lemma 3.24 (2).

Lemma 3.33. Given a Hilbert space $\mathcal{H}$ and $\varphi \in B_{\text {fp }}(\mathcal{H})$ with $i(\varphi) \leq 1$, then the following conditions are equivalent:

(1) $\varphi \circ \varphi^{*, \#}=\varphi \circ \varphi^{\#}$;

(2) $\varphi^{*, \#}=\varphi^{\dagger, d}$.

Proof. (1) $\Longrightarrow(2)$ If $\varphi \circ \varphi^{*, \#}=\varphi \circ \varphi^{\#}$, since from Lemma 2.8 we know that $\varphi^{\dagger} \circ \varphi \circ \varphi^{*}=\varphi^{*}$, we have that

$$
\varphi^{*, \#}=\varphi^{\dagger} \circ \varphi \circ \varphi^{*, \#}=\varphi^{\dagger} \circ \varphi \circ \varphi^{\#}=\varphi^{\dagger} \circ \varphi \circ \varphi^{D}=\varphi^{\dagger, d} .
$$

$(2) \Longrightarrow(1)$ Let us now assume that $\varphi^{*, \#}=\varphi^{\dagger, d}$. Then, 


$$
\varphi \circ \varphi^{*, \#}=\varphi \circ\left(\varphi^{\dagger} \circ \varphi \circ \varphi^{D}\right)=\varphi \circ \varphi^{D}=\varphi \circ \varphi^{\#} .
$$

Using similar arguments as those used in Lemma 3.33, one can easily prove that

Lemma 3.34. If $\mathcal{H}$ is a Hilbert space and $\varphi \in B_{\text {fp }}(\mathcal{H})$ with $i(\varphi) \leq 1$, one has that:

$$
\varphi^{\#, *} \circ \varphi=\varphi \circ \varphi^{\#} \Longleftrightarrow \varphi^{\#, *}=\varphi^{d, \dagger} .
$$

Lemma 3.35. Given a Hilbert space $\mathcal{H}$ and $\varphi \in B_{\text {fp }}(\mathcal{H})$ with $i(\varphi) \leq 1$, then the following conditions are equivalent:

(1) $\varphi^{\#, *}=\varphi^{*}$;

(2) $\varphi^{d, \dagger}=\varphi^{\dagger}$.

Proof. Recall now from Lemma 2.8 that $\varphi^{\dagger} \circ \varphi \circ \varphi^{*}=\varphi^{*}$ and $\varphi \circ \varphi^{*} \circ\left(\varphi^{*}\right)^{\dagger}=\varphi$.

$(1) \Longrightarrow(2)$ Let us assume that $\varphi^{\#, *}=\varphi^{*}$. Accordingly,

$$
\begin{gathered}
\varphi^{d, \dagger}=\varphi^{\#, *} \circ\left(\varphi^{*}\right)^{\dagger} \circ \varphi^{\dagger}=\varphi^{*} \circ\left(\varphi^{*}\right)^{\dagger} \circ \varphi^{\dagger}=\left(\varphi^{\dagger} \circ \varphi\right)^{*} \circ \varphi^{\dagger}=\left(\varphi^{\dagger} \circ \varphi\right) \circ \varphi^{\dagger}=\varphi^{\dagger} . \\
(2) \Longrightarrow(1) \text {. If } \varphi^{d, \dagger}=\varphi^{\dagger} \text {, then } \\
\varphi^{\#, *}=\varphi^{d, \dagger} \circ \varphi \circ \varphi^{*}=\varphi^{\dagger} \circ \varphi \circ \varphi^{*}=\varphi^{*} .
\end{gathered}
$$

Similar to Lemma 3.35 one can check that

Lemma 3.36. If $\mathcal{H}$ is a Hilbert space and $\varphi \in B_{\text {fp }}(\mathcal{H})$ with $i(\varphi) \leq 1$, one has that

$$
\varphi^{*, \#}=\varphi^{*} \Longleftrightarrow \varphi^{\dagger, d}=\varphi^{\dagger} .
$$

Proposition 3.37. Given a Hilbert space $\mathcal{H}$ and $\varphi \in B_{\text {fp }}(\mathcal{H})$ with $i(\varphi) \leq 1$, then the following conditions are equivalent:

(1) $\varphi \circ \varphi^{\#, *}=\varphi \circ \varphi^{\#}$;

(2) $\varphi^{\#, *}=\varphi^{\#}$;

(3) $\varphi \circ \varphi^{*}=\varphi \circ \varphi^{\# \text {; }}$

(4) $\varphi^{*}=\varphi^{\dagger, d}$.

Proof. Firstly, it follows from Lemma 3.18 that (1) $\Longleftrightarrow(2)$.

Moreover, since $\varphi \circ \varphi^{\#} \circ \varphi=\varphi$ and $\varphi^{\#} \circ \varphi \circ \varphi^{\#}=\varphi^{\#}$ because $i(\varphi) \leq 1$, one immediately can check that $(2) \Longleftrightarrow(3)$.

Finally, we shall prove that $(3) \Longleftrightarrow(4)$. If $\varphi \circ \varphi^{*}=\varphi \circ \varphi^{\#}$, then

$$
\varphi^{*}=\varphi^{\dagger} \circ \varphi \circ \varphi^{*}=\varphi^{\dagger} \circ \varphi \circ \varphi^{\#}=\varphi^{\dagger} \circ \varphi \circ \varphi^{D}=\varphi^{\dagger, d} .
$$

Let us now assume that $\varphi^{*}=\varphi^{\dagger, d}$. In this case, we have that

$$
\varphi \circ \varphi^{*}=\varphi \circ \varphi^{\dagger, d}=\varphi \circ \varphi^{D}=\varphi \circ \varphi^{\#} \text {. }
$$


Given an arbitrary inner product space $V$, recall now that a finite potent endomorphism $\varphi \in \operatorname{End}_{k}(V)$ admissible for the Moore-Penrose inverse is EP when $\varphi \circ \varphi^{\dagger}=\varphi^{\dagger} \circ \varphi$. It is known from [13, Lemma 3.12] that "if $\varphi$ is EP, then $i(\varphi) \leq 1$ " and, also, [13, Proposition 3.13] shows that " $\varphi$ is EP if and only if $\varphi^{\#}=\varphi^{\dagger}$.

Corollary 3.38. Let $\mathcal{H}$ be a Hilbert space and let us consider $\varphi \in B_{\text {fp }}(\mathcal{H})$ with $i(\varphi) \leq 1$. If $\varphi$ satisfies one of the equivalent conditions of Proposition 3.37, then $\varphi$ is EP.

Proof. Let us assume that $\varphi \circ \varphi^{*}=\varphi \circ \varphi^{\#}$. Hence

$$
\left(\varphi \circ \varphi^{\#}\right)^{*}=\varphi \circ \varphi^{\#}=\varphi^{\#} \circ \varphi=\left(\varphi^{\#} \circ \varphi\right)^{*}
$$

and, bearing in mind the definition of the group inverse of a finite potent endomorphism, one has that $\varphi^{\#}=\varphi^{\dagger}$ and we deduce that $\varphi$ is EP.

Proposition 3.39. If $\mathcal{H}$ is a Hilbert space and $\varphi \in B_{\text {fp }}(\mathcal{H})$ with $i(\varphi) \leq 1$, then the following conditions are equivalent:

(1) $\varphi^{*, \#} \circ \varphi=\varphi \circ \varphi^{\#}$;

(2) $\varphi^{*, \#}=\varphi^{\#}$;

(3) $\varphi^{*} \circ \varphi=\varphi \circ \varphi^{\#}$;

(4) $\varphi^{*}=\varphi^{d, \dagger}$.

Proof. Using similar arguments as those used to prove Proposition 3.37 we can check that $(1) \Longleftrightarrow(2)$ and $(2) \Longleftrightarrow(3)$. To conclude we shall prove that (3) $\Longleftrightarrow(4)$. Let us assume that $\varphi^{*} \circ \varphi=\varphi \circ \varphi^{\#}$. In this case, since $\varphi^{\#} \circ \varphi=\varphi \circ \varphi^{\#}$, one has that

$$
\varphi^{*}=\varphi^{*} \circ \varphi \circ \varphi^{\dagger}=\varphi^{\#} \circ \varphi \circ \varphi^{\dagger}=\varphi^{D} \circ \varphi \circ \varphi^{\dagger}=\varphi^{d, \dagger} .
$$

Conversely, if $\varphi^{*}=\varphi^{d, \dagger}$, then

$$
\varphi^{*} \circ \varphi=\varphi^{d, \dagger} \circ \varphi=\varphi^{D} \circ \varphi=\varphi \circ \varphi^{\#} \text {. }
$$

Analogously to the proof of Corollary 3.38, it is easy to check that

Corollary 3.40. Let $\mathcal{H}$ be a Hilbert space and let us consider $\varphi \in B_{\text {fp }}(\mathcal{H})$ with $i(\varphi) \leq 1$. If $\varphi$ satisfies one of the equivalent conditions of Proposition 3.39, then $\varphi$ is EP.

Remark 3.41. We wish to point out that, in general, the converse statements of Corollary 3.38 and Corollary 3.40 are not true. A counter-example is the following: let $\mathcal{H}$ be a 3 -dimensional Hilbert space with orthonormal basis $\left\{u_{1}, u_{2}, u_{3}\right\}$ and let us consider $\varphi \in \operatorname{End}_{\mathbb{C}}(\mathcal{H})$ such that

$$
\varphi \equiv\left(\begin{array}{lll}
1 & 3 & 0 \\
2 & 1 & 0 \\
0 & 0 & 0
\end{array}\right)
$$


in the basis $\left\{u_{1}, u_{2}, u_{3}\right\}$. We have that $\varphi$ is EP because

$$
\varphi^{\dagger}=\varphi^{\#} \equiv\left(\begin{array}{ccc}
-\frac{1}{5} & \frac{3}{5} & 0 \\
\frac{2}{5} & -\frac{1}{5} & 0 \\
0 & 0 & 0
\end{array}\right) \text {. }
$$

However,

$$
\varphi \circ \varphi^{*} \equiv\left(\begin{array}{ccc}
10 & 5 & 0 \\
5 & 5 & 0 \\
0 & 0 & 0
\end{array}\right) \neq\left(\begin{array}{ccc}
1 & 0 & 0 \\
0 & 1 & 0 \\
0 & 0 & 0
\end{array}\right) \equiv \varphi^{\#} \circ \varphi
$$

and

$$
\varphi^{*} \circ \varphi \equiv\left(\begin{array}{ccc}
5 & 5 & 0 \\
5 & 10 & 0 \\
0 & 0 & 0
\end{array}\right) \neq\left(\begin{array}{ccc}
1 & 0 & 0 \\
0 & 1 & 0 \\
0 & 0 & 0
\end{array}\right) \equiv \varphi \circ \varphi^{\#} .
$$

To finish this section we shall offer a characterization of EP bounded finite potent operators.

Proposition 3.42. If $\mathcal{H}$ is a Hilbert space and $\varphi \in B_{\text {fp }}(\mathcal{H})$ with closed $\operatorname{Im} \varphi$, then the following conditions are equivalent:

(1) $\varphi^{*}=\varphi^{*, D}$;

(2) $\varphi$ is EP;

(3) $\varphi^{*}=\varphi^{D, *}$.

Proof. (1) $\Longleftrightarrow(2)$ Let us assume that $\varphi^{*}=\varphi^{*, D}$. Then, we have that

$$
U_{\varphi} \subseteq \operatorname{Ker} \varphi^{*}=[\operatorname{Im} \varphi]^{\perp},
$$

from where we obtain that $U_{\varphi} \cap \operatorname{Im} \varphi=\{0\}$. Thus, $\operatorname{Im} \varphi=W_{\varphi}$ and $i(\varphi) \leq 1$.

Hence, from the decomposition $\mathcal{H}=\operatorname{Im} \varphi \oplus[\operatorname{Im} \varphi]^{\perp}$, we deduce that $U_{\varphi}=[\operatorname{Im} \varphi]^{\perp}=W_{\varphi}^{\perp}$ and

$$
\left(\varphi^{\dagger}\right)_{\left.\right|_{\varphi}}=0=\left(\varphi^{D}\right)_{\left.\right|_{U_{\varphi}}}=\left.\left(\varphi^{\#}\right)\right|_{\left.\right|_{\varphi}} .
$$

Moreover, since $\operatorname{Ker} \varphi=U_{\varphi}$ and $W_{\varphi}=U_{\varphi}^{\perp}$, then

$$
[\operatorname{Ker} \varphi]^{\perp}=W_{\varphi} \stackrel{\varphi}{\longrightarrow} W_{\varphi}=\operatorname{Im} \varphi
$$

and

$$
\left(\varphi^{\dagger}\right)_{\left.\right|_{W_{\varphi}}}=\left(\varphi_{\left.\right|_{W_{\varphi}}}\right)^{-1}=\left(\varphi^{D}\right)_{\left.\right|_{W_{\varphi}}}=\left(\varphi^{\#}\right)_{\left.\right|_{W_{\varphi}}} .
$$

Accordingly $\varphi^{\dagger}=\varphi^{\#}$ and, therefore, $\varphi$ is EP.

Conversely, if $\varphi$ is EP, bearing in mind that $i(\varphi) \leq 1$ and $\varphi^{\dagger}=\varphi^{\#}$, it follows from Lemma 2.8 that

$$
\varphi^{*}=\varphi^{*} \circ \varphi \circ \varphi^{\dagger}=\varphi^{*} \circ \varphi \circ \varphi^{\#}=\varphi^{*} \circ \varphi \circ \varphi^{D}=\varphi^{*, D} .
$$

$(2) \Longrightarrow(4)$ Let us now assume that $\varphi$ is EP. Since $i(\varphi) \leq 1$ and $\varphi^{\dagger}=\varphi^{\#}$, from Lemma 2.8 we deduce that

$$
\varphi^{*}=\varphi^{\dagger} \circ \varphi \circ \varphi^{*}=\varphi^{\#} \circ \varphi \circ \varphi^{*}=\varphi^{D} \circ \varphi \circ \varphi^{*}=\varphi^{D, *} .
$$


Conversely, if $\varphi^{*}=\varphi^{D \text {,* }}$, then $\operatorname{Im} \varphi^{*} \subseteq W_{\varphi}$. Thus, bearing in mind that from [14, Lemma 4.3 and Proposition 4.5] we know that $\operatorname{dim} W_{\varphi^{*}}=\operatorname{dim} W_{\varphi}$ and

$$
W_{\varphi^{*}}=U_{\varphi}^{\perp} \subseteq \operatorname{Im} \varphi^{*}
$$

we have that

$$
\operatorname{Im} \varphi^{*}=W_{\varphi}=U_{\varphi}^{\perp}=W_{\varphi^{*}}
$$

and $i(\varphi)=i\left(\varphi^{*}\right) \leq 1$.

Hence, in this case we have that $\operatorname{Im} \varphi=W_{\varphi}=U_{\varphi}^{\perp}, \operatorname{Ker} \varphi=U_{\varphi}=W_{\varphi}^{\perp}$ and, using the same arguments as above, we deduce that $\varphi^{\dagger}=\varphi^{\#}$, from where we conclude that $\varphi$ is EP.

Note that in the hypothesis of Proposition 3.42 we do not need to assume that $i(\varphi) \leq 1$.

Corollary 3.43. If $\mathcal{H}$ is a Hilbert space and $\varphi \in B_{\text {fp }}(\mathcal{H})$ with $i(\varphi) \leq 1$, then the following conditions are equivalent:

(1) $\varphi^{\dagger}=\varphi^{\dagger, d}$;

(2) $\varphi^{*}=\varphi^{*, \#}$;

(3) $\varphi$ is EP;

(4) $\varphi^{*}=\varphi^{\#, *}$;

(5) $\varphi^{\dagger}=\varphi^{d, \dagger}$.

Proof. The assertions are immediately deduced from Lemmas 3.35, 3.36 and Proposition 3.42.

Remark 3.44. Let $A \in \operatorname{Mat}_{n \times n}(\mathbb{C})$ be an $n \times n$ matrix with entries in the complex numbers. If $i(A) \leq 1$, then Corollary 3.43 shows that the following conditions are equivalent:

(1) $A^{\dagger}=A^{\dagger, D}$;

(2) $A^{*}=A^{*, \#}$;

(3) $A$ is $\mathrm{EP}$;

(4) $A^{*}=A^{\#, *}$;

(5) $A^{\dagger}=A^{D, \dagger}$.

\section{Applications to Linear Systems on Hilbert Spaces of the Drazin-Star Inverse}

Finally, similar to the statements of [10, Section 4], we shall apply the above properties of the Drazin-Star inverse of bounded finite potent endomorphisms for studying the consistence and the general solutions of linear systems on Hilbert spaces. 
Lemma 4.1. Given a Hilbert space $\mathcal{H}$ and an endomorphism $\varphi \in B_{\text {fp }}(\mathcal{H})$ with closed $\operatorname{Im} \varphi$ and $i(\varphi)=r$, then

$$
\operatorname{Ker} \varphi^{l}=\operatorname{Im}\left(I d-\varphi^{D} \circ \varphi\right) \quad \text { for all } l \geq r .
$$

Proof. If $\mathcal{H}=W_{\varphi} \oplus U_{\varphi}$ is the AST-decomposition of $\mathcal{H}$ induced by $\varphi$, it is clear that

$$
\operatorname{Ker} \varphi^{l}=U_{\varphi}=\operatorname{Im}\left(\operatorname{Id}-\varphi^{D} \circ \varphi\right) \quad \text { for all } l \geq r,
$$

and the assertion is proved.

Proposition 4.2. If $\mathcal{H}$ is a Hilbert space and $\varphi \in B_{f p}(\mathcal{H})$ with closed $\operatorname{Im} \varphi$ and $i(\varphi)=r$, then the linear system

$$
\varphi^{l}(x)=\varphi^{l}\left(\varphi^{*}(h)\right)
$$

is consistent for each $h \in \mathcal{H}$ and every $l \geq r$ and its general solution is

$$
x=\varphi^{D, *}(h)+\operatorname{Im}\left(I d-\varphi^{D} \circ \varphi\right)
$$

or, equivalently,

$$
x=\varphi^{D, *}(h)+U_{\varphi} .
$$

Proof. It follows from Lemma 4.1 that for checking the claim of this proposition is sufficient with proving that $\varphi^{D, *}(h)$ is a particular solution of (4.1).

Accordingly, bearing in mind that

$$
\varphi^{l}\left(\varphi^{D, *}(h)\right)=\left(\varphi^{l} \circ \varphi^{D} \circ \varphi\right)\left(\varphi^{*}(h)\right)=\varphi^{l}\left(\varphi^{*}(h) \quad \text { for all } l \geq r,\right.
$$

the statement is deduced.

A direct consequence of Lemma 4.1 and Proposition 4.2 is:

Corollary 4.3. If $\mathcal{H}$ is a Hilbert space, $\varphi \in B_{\text {fp }}(\mathcal{H})$ with closed $\operatorname{Im} \varphi$ and $i(\varphi)=$ $r$ and $\mathcal{H}=W_{\varphi} \oplus U_{\varphi}$ is the $A S T$-decomposition of $\mathcal{H}$ induced by $\varphi$, then

$$
\varphi^{D, *}(h)-\varphi^{*}(h) \in U_{\varphi}
$$

for all $h \in \mathcal{H}$.

Example 4.4. If $\mathcal{H}$ is a separable Hilbert space with orthonormal basis $\left\{u_{i}\right\}_{i \in \mathbb{N}}$ and $\varphi \in B_{f p}(\mathcal{H})$ is the endomorphism defined in Example 3.26 with $i(\varphi)=2$, we have that the equation $\varphi^{4}(x)=\varphi^{4}\left(\varphi^{*}(h)\right)$ is equivalent to the linear system

$$
\begin{aligned}
12 x_{1}+9 x_{2}-15 x_{3} & =15 h_{1}+42 h_{2}-36 h_{3}+42 h_{4} \\
-2 x_{1}-12 x_{2}-35 x_{3} & =-61 h_{1}+68 h_{2}-117 h_{3}+68 h_{4} \\
13 x_{1}+24 x_{2}+34 x_{3} & =95 h_{1}-55 h_{2}+126 h_{3}-55 h_{4},
\end{aligned}
$$

where $x=\left(x_{i}\right)$ and $h=\left(h_{i}\right)$ are the coordinates expressions of $x$ and $h$ in $\left\{u_{i}\right\}_{i \in \mathbb{N}}$ respectively. 
Accordingly, since $U_{\varphi}=\overline{\left\langle u_{i}\right\rangle_{i \geq 4}}$, a non-difficult computation shows that the general solution of $(4.2)$ is

$$
\begin{aligned}
& x_{1}=h_{1}+h_{2}+h_{4} \\
& x_{2}=2 h_{1}+h_{3} \\
& x_{3}=h_{1}-2 h_{2}+3 h_{3}-2 h_{4} \\
& x_{i}=\lambda_{i} \text { for } i \geq 4
\end{aligned}
$$

with $\sum_{i \geq 4}\left|\lambda_{i}\right|<\infty$.

Lemma 4.5. Given a Hilbert space $\mathcal{H}$ and an endomorphism $\varphi \in B_{\text {fp }}(\mathcal{H})$ with closed $\operatorname{Im} \varphi$ and $i(\varphi)=r$, if $\mathcal{H}=W_{\varphi} \oplus U_{\varphi}$ is the AST-decomposition of $\mathcal{H}$ induced by $\varphi$, then

$$
\operatorname{Ker}\left(\varphi^{\dagger}\right)^{*} \cap W_{\varphi}=\{0\}
$$

Proof. If $\mathcal{H}=W_{\varphi^{*}} \oplus U_{\varphi^{*}}$ is the AST-decomposition of $\mathcal{H}$ induced by $\varphi^{*}$, since from [14, Proposition 4.5] one has that $W_{\varphi^{*}}=U_{\varphi}^{\perp}$ and from Proposition 2.7 we know that $\left(\varphi^{*}\right)^{\dagger}=\left(\varphi^{\dagger}\right)^{*}$, one has that

$$
\operatorname{Ker}\left(\varphi^{\dagger}\right)^{*}=\operatorname{Ker}\left(\varphi^{*}\right)^{\dagger}=\left[\operatorname{Im} \varphi^{*}\right]^{\perp} \subseteq U_{\varphi},
$$

because $U_{\varphi}^{\perp} \subseteq \operatorname{Im} \varphi^{*}$ and $U_{\varphi}$ is closed.

Accordingly, $\operatorname{Ker}\left(\varphi^{\dagger}\right)^{*} \cap W_{\varphi}=\{0\}$ and the claim is proved.

Proposition 4.6. Let $\mathcal{H}$ be a Hilbert space, let $\varphi \in B_{\text {fp }}(\mathcal{H})$ with closed $\operatorname{Im} \varphi$ and $i(\varphi)=r$ and let $\tilde{h} \in \operatorname{Im}\left(\left(\varphi^{\dagger}\right)^{*} \circ \varphi^{D}\right)$. Then, $\varphi^{D, *}(\tilde{h})$ is the unique solution in $W_{\varphi}$ of the linear system

$$
\left(\varphi^{\dagger}\right)^{*}(x)=\tilde{h} .
$$

Proof. If $\left.\tilde{h}=\left(\varphi^{\dagger}\right)^{*}\right)\left(\varphi^{D}\left(h^{\prime}\right)\right)$ for a certain $h^{\prime} \in \mathcal{H}$, since from Corollary 3.11 one can check that

$$
\varphi^{D} \circ \varphi \circ \varphi^{\dagger} \circ \varphi \circ \varphi^{D}=\varphi^{D},
$$

and bearing in mind that

$$
\left.\left(\varphi^{\dagger}\right)^{*}\left(\varphi^{D, *}(\tilde{h})\right)=\left(\left(\varphi^{\dagger}\right)^{*} \circ\left(\varphi^{D} \circ \varphi \circ \varphi^{*}\right) \circ\left(\varphi^{\dagger}\right)^{*}\right)\left(\varphi^{D}\left(h^{\prime}\right)\right)=\left(\varphi^{\dagger}\right)^{*}\right)\left(\varphi^{D}\left(h^{\prime}\right)\right)=\tilde{h},
$$

the assertion is immediately deduced from Lemma 4.5 because

$$
\varphi^{D, *}(\tilde{h}) \in \operatorname{Im} \varphi^{D}=W_{\varphi} .
$$

Corollary 4.7. Let $\mathcal{H}$ be a Hilbert space, let $\varphi \in B_{\text {fp }}(\mathcal{H})$ with $i(\varphi) \leq 1$ and let $\tilde{h} \in \operatorname{Im} \varphi$. Then, $\varphi^{D, *}(\tilde{h})$ is the unique solution in $\operatorname{Im} \varphi$ of the linear system

$$
\left(\varphi^{\dagger}\right)^{*}(x)=\tilde{h} .
$$


Proof. With the above notation, the claim is a direct consequence of Proposition 4.6 because, when $i(\varphi) \leq 1$, it follows from Proposition 2.7 and [14, Proposition 4.3] that

$$
\operatorname{Im}\left(\varphi^{\dagger}\right)^{*}=\operatorname{Im}\left(\varphi^{*}\right)^{\dagger}=\left[\operatorname{Ker}\left(\varphi^{*}\right)\right]^{\perp}=\left[U_{\varphi^{*}}\right]^{\perp}=W_{\varphi}=\operatorname{Im} \varphi
$$

and, therefore, $\operatorname{Im}\left(\left(\varphi^{\dagger}\right)^{*} \circ \varphi^{D}\right)=\operatorname{Im} \varphi$.

Example 4.8. Keeping again the data of Example 3.26, from an easy computation one has that

$\operatorname{Im}\left(\left(\varphi^{\dagger}\right)^{*} \circ \varphi^{D}\right)=<14 u_{1}-20 u_{2}-19 u_{3},-20 u_{1}+35 u_{2}+31 u_{3},-19 u_{1}+31 u_{2}+29 u_{3}>$.

Accordingly, given

$$
\tilde{h}=(14 \lambda-20 \mu-19 \delta) u_{1}+(-20 \lambda+35 \mu+31 \delta) u_{2}+(-19 \lambda+31 \mu+29 \delta) u_{3}
$$

with $\lambda, \mu, \delta \in \mathbb{C}$, one has that the system $\left(\varphi^{\dagger}\right)^{*}(x)=\tilde{h}$ is determined by the equations

$$
\begin{aligned}
-\frac{2}{3} x_{1}+x_{2}-\frac{1}{3} x_{3} & =14 \lambda-20 \mu-19 \delta \\
\frac{5}{3} x_{1}-x_{2}+\frac{1}{3} x_{3} & =-20 \lambda+35 \mu+31 \delta \\
\frac{4}{3} x_{1}-x_{2}+\frac{2}{3} x_{3} & =-19 \lambda+31 \mu+29 \delta
\end{aligned}
$$

where $x=\left(x_{i}\right) \in \mathcal{H}$. Thus, from Proposition 4.6 one has that

$$
\begin{aligned}
& x_{1}=-6 \lambda+15 \mu+12 \gamma \\
& x_{2}=9 \lambda-9 \mu-9 \gamma \\
& x_{3}=-3 \lambda+3 \mu+6 \gamma \\
& x_{4}=9 \lambda-9 \mu-9 \gamma \\
& x_{5}=0 \text { for all } i \geq 5
\end{aligned}
$$

is the unique solution in $W_{\varphi}=\left\langle u_{1}, u_{2}+u_{4}, u_{3}\right\rangle$ of (4.3).

\section{Acknowledgements}

The author would like to thank Dr. D. Mosić for her useful comments about a preliminary version of this paper. Moreover, the author thanks the anonymous reviewer for his/her valuable comments to improve the quality of the paper.

Funding Open Access funding provided thanks to the CRUE-CSIC agreement with Springer Nature. This work is partially supported by the Spanish Government research Projects No. PGC2018-099599-B-I00 and the Regional Government of Castile and Leon research Project No. J416/463AC03. 
Open Access. This article is licensed under a Creative Commons Attribution 4.0 International License, which permits use, sharing, adaptation, distribution and reproduction in any medium or format, as long as you give appropriate credit to the original author(s) and the source, provide a link to the Creative Commons licence, and indicate if changes were made. The images or other third party material in this article are included in the article's Creative Commons licence, unless indicated otherwise in a credit line to the material. If material is not included in the article's Creative Commons licence and your intended use is not permitted by statutory regulation or exceeds the permitted use, you will need to obtain permission directly from the copyright holder. To view a copy of this licence, visit http://creativecommons. org/licenses/by/4.0/.

\section{References}

[1] Argerami, M., Szechtman, F., Tifenbach, R.: On Tates trace. Linear Multilinear Algebra 55(6), 515-520 (2007)

[2] Braunling, O.: Adele residue symbol and Tates central extension for multiloop Lie algebras. Algebra Number Theory 8(1), 19-52 (2014)

[3] Braunling, O.: On the local residue symbol in the style of Tate and Beilinson. N. Y. J. Math. 2(4), 458-513 (2018)

[4] Cabezas Sánchez, V., Pablos Romo, F.: Explicit solutions of infinite systems of linear equations from reflexive generalized inverses of finite potent endomorphisms. Linear Algebra Appl. 559, 125-144 (2018)

[5] Cabezas Sánchez, V., Pablos Romo, F.: Moore-Penrose inverse of some linear maps on infinite-dimensional vector spaces. Electron. J. Linear Algebra 36, 570586 (2020)

[6] Campbell, S.L., Meyer Jr: Generalized Inverses of Linear Transformations. Dover (1991). ISBN978-0-486-66693-8

[7] Debry, C.P.: Towards a class number formula for Drinfeld modules. University of Amsterdam (2016)

[8] Drazin, M.P.: Pseudo-inverses in associative rings and semigroups. Am. Math. Mon. 65(7), 506-514 (1958)

[9] Hernández Serrano, D., Pablos Romo, F.: Determinants of finite potent endomorphisms, symbols and reciprocity laws. Linear Algebra Appl. 469, 239-261 (2013)

[10] Mosić, D.: Drazin-Star and Star-Drazin matrices. Results Math. 75, 61 (2020). https://doi.org/10.1007/s00025-020-01191-7

[11] Pablos Romo, F.: Core-Nilpotent decomposition and new generalized inverses of finite potent endomorphisms. Linear Multilinear Algebra 68(11), 2254-2275 (2020)

[12] Pablos Romo, F.: Generalized Inverses of Bounded Finite Potent Operators on Hilbert Spaces, Preprint. University of Salamanca (2021)

[13] Pablos Romo, F.: Group inverse of finite potent endomorphisms on arbitrary vector spaces. Oper. Matrices 14(4), 1029-1042 (2020) 
[14] Pablos Romo, F.: On bounded finite potent endomorphisms on arbitrary Hilbert spaces. Bull. Malays. Math. Sci. Soc. 44(6), 4085-4107 (2021). https://doi.org/ 10.1007/s40840-021-01156-1

[15] Pablos Romo, F.: On Drazin-Moore-Penrose inverses of finite potent endomorphisms. Linear Multilinear Algebra 69(4), 627-647 (2021)

[16] Pablos Romo, F.: On the Drazin inverse of finite potent endomorphisms. Linear Multilinear Algebra 67(10), 2135-2146 (2019)

[17] Pablos Romo, F.: On the linearity property of Tates trace. Linear Multilinear Algebra 55(4), 523-526 (2007)

[18] Ramos González, J., Pablos Romo, F.: A negative answer to the question of the linearity of Tates trace for the sum of two endomorphisms. Linear Multilinear Algebra 62(4), 548-552 (2014)

[19] Taelman, L.: Special L-values of Drinfeld modules. Ann. Math. 175(1), 369-391 (2012)

[20] Tate, J. Residues of differentials on curves. Ann. Scient.Éc. Norm. Sup. 1, 149159 (1968)

[21] Yekutieli, A.: Local Beilinson-Tate operators. Algebra Number Theory 9(1), 173-224 (2015)

Fernando Pablos Romo

Departamento de Matemáticas

Universidad de Salamanca

Plaza de la Merced 1-4

37008 Salamanca

Spain

e-mail: fpablos@usal.es

Received: May 17, 2021.

Accepted: October 13, 2021.

Publisher's Note Springer Nature remains neutral with regard to jurisdictional claims in published maps and institutional affiliations. 